\title{
Multiple scales in streamer discharges, with an emphasis on moving boundary approximations
}

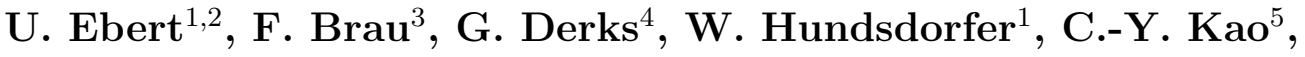

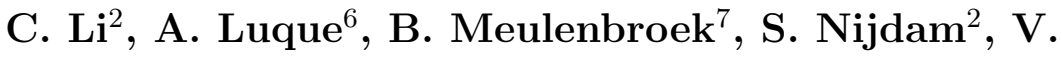 \\ Ratushnaya $^{1}$, L. Schäfer ${ }^{8}$, S. Tanveer ${ }^{5}$ \\ ${ }^{1}$ CWI Amsterdam, P.O.Box 94079, 1090GB Amsterdam, The Netherlands, \\ ${ }^{2}$ Faculty of Applied Physics, Technische Universiteit Eindhoven, The Netherlands, \\ ${ }^{3}$ Groupe de Physique Nucleaire Théorique, Université de Mons-Hainaut, Belgium, \\ ${ }^{4}$ Department of Mathematics, University of Surrey, UK, \\ ${ }^{5}$ Department of Mathematics, Ohio State University, USA, \\ ${ }^{6}$ Institito de Astrofisica de Andalucia, CSIC, Granada, Spain, \\ ${ }^{7}$ Institute of Applied Mathematics, Technische Universiteit Delft, The Netherlands, \\ ${ }^{8}$ Fachbereich Physik, Universität Duisburg-Essen, Germany. \\ E-mail: ebert@cwi.nl
}

\begin{abstract}
Streamer discharges determine the very first stage of sparks or lightning, and they govern the evolution of huge sprite discharges above thunderclouds as well as the operation of corona reactors in plasma technology. Streamers are nonlinear structures with multiple inner scales. After briefly reviewing basic observations, experiments and the microphysics, we start from density models for streamers, i.e., from reaction-drift-diffusion equations for charged particle densities coupled to the Poisson equation of electrostatics, and focus on derivation and solution of moving boundary approximations for the density models. We recall that so-called negative streamers are linearly stable against branching, and that streamer groups in two dimensions are well approximated by the classical Saffman-Taylor finger of two fluidflow. We draw conclusions on streamer physics, and we identify open problems in the moving boundary approximations.
\end{abstract}




\section{Discharges in laboratory and in nature, and their multiple scales}

\subsection{Branching streamer trees in the laboratory}

When a high voltage is applied to a sufficiently large volume of ionizable matter, a discharge typically paves its way in the form of a tree with growing branches. The title page of Nonlinearity in 2011 shows the false color image of such a discharge, taken from [1]; the figure is reproduced in Fig. 1. The discharge here propagates through argon at standard temperature and pressure; at the upper middle of the picture, an invisible electrode needle supplies a positive voltage pulse of $22 \mathrm{kV}$, and the photograph integrates the light emission over about a microsecond. The height shown is about 4 $\mathrm{cm}$, while the distance from the needle to a lower planar electrode is $16 \mathrm{~cm}$.

The ionized branches that penetrate the ionized matter, are called streamers. They are formed by the following mechanism: (i) if free electrons are accelerated by sufficiently high local electric fields, they can create additional electron-ion pairs by impact and in this manner generate an ionization avalanche; (ii) the light electrons rapidly drift in the electric field away from the positive ions, and therefore electrons and ions form space charges; (iii) the space charges modify the local electric field. A streamer branch or finger is characterized by a strong field suppression in its ionized interior and by a consecutive strong field enhancement at its curved tip. This local field enhancement creates a region with strong local impact ionization at the streamer tip that allows the streamer to penetrate into regions where the background field would be too low for ionization. Accordingly, the streamer is a strongly nonlinear object. Within the sequence of events that lead to electric breakdown in sparks and lightning, it is the earliest stage, and it is the state furthest from equilibrium as no heating supports the existence of a plasma state yet.

Streamers come in two polarities: If they emerge from a negatively charged electrode, they carry a net negative charge and move in the same direction as electrons drift. If they emerge from a positively charged electrode, they carry a net positive charge and move against the electron drift direction.

The title picture was chosen for its beauty. We interpret the feathery structure as avalanches initiated by single electrons and propagating towards the positive streamer $[1,2]$. Obviously, a full understanding of these structures requires a stochastic approach to the single electron dynamics as recently developed in Monte Carlo and hybrid models $[3,4,5,6,7,8]$, but these microscopic features will be discussed elsewhere.

In the present article we will concentrate on much smoother structures as they appear, e.g., in discharges in air. Positive streamers in air are shown in Figs. 2 and 3. These discharges do not show obvious traces of single electron stochastics for reasons discussed in section 2.2. The left column in Fig. 2 (panels (a), (b), (c)) shows positive streamer discharge trees; a voltage pulse of $35 \mathrm{kV}$ is applied for $130 \mathrm{~ns}$ to air at standard temperature and pressure; and $5 \mathrm{~cm}$ out of the full gap height of $16 \mathrm{~cm}$ are shown. The panel (a) shows a time integrated picture just like Fig. 1; the streamers propagate as long as the voltage pulse of 130 ns duration supports them. Panels (b) and (c) are 
taken with an exposure time of $30 \mathrm{~ns}$ and a delay of 0 or $60 \mathrm{~ns}$ with respect to the application of the voltage pulse; they show the light emission during an early and a late stage of the dynamics. The intensity levels are color coded in the same manner in panels (a) and (b), but in panel (c) the discharge emits less light, and the same color represents lower intensities. Panels (b) and (c) show that not the whole channel emits light, but only the pieces of the channel that grow during the exposure time of the camera. Another pedagogical illustration of the effect that only the actively growing tips of the channels emit light, can be found in Fig. 1 in [9]. Adjusting the opening and exposure time of the ICCD camera therefore has become an important tool in analyzing streamer dynamics $[10,11,12,13,14,15,16]$. With exposure times as short as $300 \mathrm{ps}$, even the shell type inner structure of the ionization front within the streamer tip could be reconstructed [17]. This structure corresponds to what is expected from simulations, as we will discuss in section 1.3.

The streamer trees are three-dimensional objects while a normal photograph only shows a two-dimensional projection. This three-dimensional structure can already be seen in Fig. 1 as some branches are out of focus. Fig. 3 shows how the three-dimensional structure can be recovered through stereoscopic imaging. The method is introduced in [18] to measure branching angles; it is improved and used as well in [19] to analyze whether and when streamer channels really reconnect and when they just pass behind each other.

The right column in Fig. 2 (panels (d), (e), (f)) finally zooms into the emergence of positive streamers in air at the electrode tip. It shows how the discharge starts as a homogeneously glowing ball at the tip (d). It then expands into a shell-shaped front (e). This front destabilizes and emits streamers (f). The next stage of evolution is not shown in in the right column of Fig. 2 anymore, as one now needs to zoom out, and the appearance will then be similar to panel (b) in Fig. 2.

A negative discharge forms a very similar ball and subsequent shell-shaped front as the positive discharge in panels (d) and (e) of Fig. 2. But this shell expands further and does not destabilize so early into streamers. If the negative voltage pulse lasts for only $130 \mathrm{~ns}$ in the experimental set-up as described above, the shell-shaped front does not destabilize at all.

\subsection{Occurrence of streamers in nature and technology}

Streamers form the first stage of sparks and lightning, and the stage that is furthest from equilibrium. They explore large parts of the non-ionized medium by their multiply branched tree-like structure. In a lightning stroke, the streamer corona paves the way for a hot lighting leader that maintains the created conducting path through Ohmic heating. Lightning leaders also explore different paths, but less than their streamer coronas. Once a conducting path is formed between two charged cloud regions or between cloud and ground, the lightning return stroke chooses mostly only one of the available channels the winner takes all —; this return stroke appears as the visible lightning stroke. 
But the streamers also appear alone without these subsequent processes. On the one hand, they are used in many technical processes; the benefit is that electric energy is directly used for electron acceleration and consecutive excitations and reactions of the gas molecules, while essentially no energy is wasted for general gas heating. Modern efficient streamer corona reactors are based on supplying a voltage pulse only as long as the streamer propagation phase lasts. On the other hand, the gigantic sprite discharges above thunderclouds as illustrated in Fig. 4 are now recognized as being physically similar to the small streamer discharges from Figs. 1-3. The similarity relation between streamers in different gas densities, the so-called Townsend scaling, is easily established by dimensional analysis; it is discussed in detail in [20] and briefly recalled in section 2.1. Further recent reviews on streamer discharges in nature and technology can be found in Plasma Sources Sci. Technol. [9] in 2006, in a cluster issue on "Streamers, Sprites and Lightning" in J. Phys. D [21] in 2008, and in J. Geophys. Res. [20] in 2010.

\subsection{Underlying physical mechanisms on multiple scales}

Streamer modeling starts on the level of collisions of electrons with molecules. Electrons are accelerated in the local field and collide with neutral molecules. If they have gained sufficient energy, they can excite or even ionize the gas molecules, and loose part of their energy during this process. If the collision is ionizing, the molecule is split into a new electron ion pair and the local conductivity increases. As the molecule density is always much larger than the density of electrons and ions, the neutral background gas is treated as a random background through which the electrons move, loosing and gaining energy. The electron motion is typically modeled by a Monte Carlo model. An additional challenge (of plasma physics in general) lies in identifying appropriate differential cross-sections for the particle collisions in different gases. The derivation of a density or PDE approximation for the electron and ion density from the stochastic Monte Carlo model is also an art in itself [3, 6, 8, 22, 23, 24, 25]. In the present article, we focus on nonlinear dynamics aspects from the PDE level upwards.

An essential element of streamer dynamics both on the particle and on the density level is that the electrons drift in the electric field, create space charges and hence modify the background field. The inner structure of a positive streamer in air in a density approximation simulation is shown in Fig. 5. The exterior is non-ionized. Inside the streamer, the densities of electrons $n_{e}$ and ions $n_{+}$are essentially equal, but along its surface, there is a net charge $q \propto n_{+}-n_{e}$ concentrated in a very thin layer. This charge layer strongly modifies the electric field: it is small (and nearly homogeneous) in the interior and strongly enhanced at the streamer tip. In this self-created region of strongly enhanced field, the ionization reaction is very efficient and the streamer grows. It is also this active tip region that emits the light that is observed in the experimental Figs. 1-4.

In the present paper, we discuss the density approximations for streamer models in section 2. Section 3 is devoted to experimental and numerical evidence of streamer 
branching, and to the properties of planar streamer ionization fronts with an emphasis on their pulled dynamics on the one hand and on their dynamical instability against transversal perturbations on the other hand. In section 4, the structure of a moving boundary approximation for the streamer ionization fronts is sketched and the boundary condition is derived. Section 5 summarizes solutions of the moving boundary problem for compact streamers in two dimensions and draws conclusions on streamer branching. Section 6 contains results on the relation with Saffman-Taylor fingers, and on field enhancement at the tips of streamer groups. For further steps towards quantitative models of complete streamer trees, we refer to future work [26].

\section{Density models for streamers}

\subsection{Minimal streamer model and corrections}

The minimal PDE model for streamers [27, 28, 9] consists of a reaction-drift-diffusion equation for the electron density $\sigma$ and a reaction equation for the density $\rho$ of positive ions (whose mobility is neglected) coupled to the electrostatic field $\mathbf{E}=-\nabla \varphi$. It applies to so-called non-attaching gases like nitrogen and argon that do not form negative ions. The model reads in dimensionless units

$$
\begin{aligned}
\partial_{t} \sigma & =D \nabla^{2} \sigma+\nabla \cdot(\sigma \mathbf{E})+\sigma|\mathbf{E}| \alpha(|\mathbf{E}|), \\
\partial_{t} \rho & =\sigma|\mathbf{E}| \alpha(|\mathbf{E}|), \\
\nabla \cdot \mathbf{E} & =\rho-\sigma, \quad \mathbf{E}=-\nabla \varphi .
\end{aligned}
$$

Here $D$ is the dimensionless electron diffusion constant; Townsend's ionization coefficient $\alpha(E)$ is a positive function that vanishes for vanishing field. A much used approximation is $\alpha(E)=e^{-1 /|E|}$ in dimensionless units; it goes back to Townsend about 100 years ago. The model describes negative discharges that propagate in the drift direction of electrons; for numerical solutions of the model for single propagating streamers, we refer to $[29,30,31,32]$. The physical units in nitrogen $[27,28,9]$ are approximately $n_{e}=\sigma \cdot 5 \cdot 10^{14} / \mathrm{cm}^{3}\left(N / N_{0}\right)^{2}$ for the electron number density, $n_{+}=\rho \cdot 5 \cdot 10^{14} / \mathrm{cm}^{3}\left(N / N_{0}\right)^{2}$ for the ion number density, $\mathbf{E}_{\text {phys }} \approx \mathbf{E}_{\text {dim-less }} * 200 \mathrm{kV} / \mathrm{cm}\left(N / N_{0}\right)$ for the electric field, and dimensionless times and lengths are multiples of $3 \mathrm{ps}\left(N_{0} / N\right)$ and $2.3 \mu \mathrm{m}\left(N_{0} / N\right)$. The diffusion constant is $D=0.1$. Here $N$ is the gas particle number density, and $N_{0}$ is the same at standard temperature and pressure, i.e., the dimensional parameters directly show the scaling relations between similar discharges at different gas densities $N / N_{0}$. This Townsend scaling relates small laboratory discharges (in Figs. 1-3) to huge sprite discharges (in Fig. 4); range of validity and corrections to Townsend scaling is discussed in detail in [20].

While this model is convenient for qualitative understanding and analytical approximations, much knowledge has been gained in recent years on the actual microphysics. Differential cross-sections for the various elastic, inelastic and ionizing scattering processes during collisions of electrons with neutral molecules or atoms have been collected, and mobilities, diffusion coefficients and reaction rates have been 
calculated as a function of the electric field $[3,6,8]$. This introduces quantitative, but no qualitative corrections to the minimal model above.

\subsection{Electron runaway}

However, at electric fields above $260 \mathrm{kV} / \mathrm{cm}\left(N / N_{0}\right)$ in nitrogen and air, the density model breaks down, because the electrons do not relax to some steady state between energy gain through field acceleration and energy loss during collisions, but they continuously gain more energy than they loose so that they keep accelerating and run away. Electron run-away from streamers is currently investigated as a possible cause of hard radiation from laboratory discharges and of terrestrial gamma-ray flashes from thunderstorms $[33,3,4,5,7]$.

\subsection{Extensions of the density model for streamers in air}

For streamers in air, the density model has to be extended, also in moderate fields. First, the oxygen in air is an attaching gas, i.e., it captures free electrons and forms negative ions. Electron attachment dominates over the impact ionization reaction below the so-called break-down field $E_{k}$. Effectively, this means that the reaction coefficient $\alpha$ is negative for $0<E<E_{k}$ and positive for $E>E_{k}$. As long as the negative ions are approximated as immobile, they can be absorbed into an effective ion density $\rho=\rho_{+}-\rho_{-}$, where $\rho_{ \pm}$are the number densities of positive and negative ions, respectively. No new equation needs to be introduced.

Second, nitrogen-oxygen-mixtures like air have a nonlocal ionization reaction mediated by photons, the so-called photo-ionization discussed, e.g., in $[34,1,2]$. In ambient air, photo-ionization introduces an ionization length of about $1.3 \mathrm{~mm}$. Numerical solutions of single positive streamers in ambient air including photo-ionization can be found, e.g., in [34, 35, 36, 37, 38, 39, 40, 12, 41, 42, 43, 44, 45, 46].

Electron attachment and photo-ionization are two independent processes that both become important when oxygen is added to nitrogen, but is in general uncorrelated in other gas mixtures. However, there is little recent experimental research on streamers in other gases than in air, see $[1,2,47]$ and references therein.

\subsection{Streamers of positive and negative polarity}

It is a general experimental observation that positive streamers in air emerge much easier than negative ones, and air is the most investigated gas. Therefore there is a tendency in the literature to take the term streamer as equivalent to positive streamers in air, i.e., these streamers propagate against the electron drift and are supported by photo-ionization.

However, recent progress in electrical engineering has supplied us with electric circuits where the voltage can rise to the order of $100 \mathrm{kV}$ within nanoseconds. If voltage 
rise times are below $200 \mathrm{~ns}$ and voltages above $40 \mathrm{kV}$, positive and negative streamers become increasingly similar $[13,15]$.

On the theoretical side, we now understand a number of features about the difference between positive and negative streamers in air and in other gases:

(i) Negative streamers in air in undervolted gaps (where the background field is below the breakdown value) propagate quite as if photo-ionization was absent, while photoionization largely accelerates them in higher fields [34, 43].

(ii) Negative streamers in air propagate slower than positive streamers, both in experiments $[13,15]$ and in simulations [43]. This seems paradoxical as the negative streamer is helped by electron drift in its motion while the positive streamer is hindered. The explanation of this surprising fact lies in the observation that a negative streamer channel becomes wider along its whole length due to electrons drifting outwards. Therefore the electric field at the tip of a negative streamer is less focussed and enhanced than in a positive streamer, and the positive streamer ultimately is faster [43].

(iii) Positive streamers do not require photo-ionization for propagation. Experiments in nitrogen with less than $1 \mathrm{ppm}$ oxygen were aimed at suppressing photo-ionization, but still showed propagating positive streamers [1]. As we understand now [48, 2], even an oxygen admixture of $10^{-6}$ still supports streamer propagation by photo-ionization in almost pure nitrogen, but the background ionization due to natural radioactivity or due to previous discharges can support a very similar propagation mode.

\section{Streamer branching and the stability properties of ionization fronts}

\subsection{Experimental characterization of branching streamers}

As discussed in section 1.1 and demonstrated in Figs. 1-3, streamers branch frequently. Branching is the key feature in forming the streamer tree. In experiments, the branching structure of positive streamers in air was recently investigated, first in two-dimensional images [16], then in full three-dimensional stereoscopic reconstructions [18]. The available experiments show that the ratio between propagation length until branching and streamer diameter has an approximately constant value of 15 , independently of the streamer diameter, and that the branching angles are approximately Gaussian distributed with a mean of $43^{\circ}$ and a standard deviation of $12^{\circ}$; these angles do not significantly depend on the distance from the needle or on the gas pressure [18]. In experiments at pressures of 50 to 1000 mbar, streamer channels always branch in not more than two new branches, while in sprite discharges also many more branches are seen $[49,50,51]$.

There are no quantitative predictions yet for branching positive streamers in air, but branching negative streamers in nitrogen have been studied. We now review the state of this theory. 


\subsection{Numerical evidence of streamer branching}

According to oral accounts, branching streamers were seen in a number of numerical simulations of density models. But these observations were typically discarded as numerical errors. Kulikovsky [37] published figures where a streamer lost its convexity and propagated as a hollow channel; he interpreted this as streamer branching. However Pancheshnyi and Starikovskii [52] pointed to numerical problems in the calculation of photo-ionization and showed figures of stable streamer propagation under the same conditions.

The reluctance to investigate branching using deterministic numerical models is probably related to the fact that a streamer branching concept already existed. It dates back to the 1930'es and is illustrated in many textbooks while it has never been verified. The concept is discussed and criticized in more detail in [9]. It supposes that the long range photo-ionization in air creates few ionization avalanches at random places ahead of the streamer. These avalanches immediately form new branches when they approach the streamer body. However, the avalanches are now indeed visible in very pure gases [1], and their density is estimated in [2], but neither do these avalanches lead to immediate branching in pure gases nor would they explain streamer branching in air, because they are so numerous in air that individual avalanches cannot be distinguished. Rather they have a stabilizing effect in air, see below.

When Arrayás et al. found branching negative streamers in density models in nitrogen, they interpreted their results as a Laplacian instability [53] that could occur in fully deterministic models. This statement was motivated by similar instabilities in other pattern forming systems like two-fluid flow in Hele-Shaw cells [54], solidification fronts, growth of bacterial colonies etc. [27, 28, 55]. A second simulation with the streamer detached from an electrode and branching in a similar manner was published in [56]. The results were criticized [57] as possibly numerically erroneous, as constrained to cylindrical symmetry, and as not addressing "the real problem" of positive streamers in air with nonlocal photo-ionization. The authors replied in [58]; in particular, the cylindrical symmetry suppressed all modes without this symmetry, therefore the branching time with symmetry constraint gave an upper bound to the branching time without constraint.

4 years later, possible numerical errors in the branching of negative streamers in nitrogen were excluded by a new code with adaptive grid refinement, but still with cylindrical symmetry. Results are shown in Fig. 6. The numerical method is described in [59]. The paper [60] showed that the branching time of negative streamers in nitrogen converged when the numerical grid was sufficiently fine. A numerical approach to the full three-dimensional problem was developed in [61]. Numerical results on negative streamer branching in three dimensions were derived only recently; they are now submitted [62] and show that indeed the branching time with symmetry constraint is a good approximation to branching in the full three-dimensional density model.

However, the photo-ionization reaction in air stabilizes the solutions of density 
models, and suppresses branching. This was found for both positive and negative streamers without grid refinement in [40] and for negative streamers with grid refinement in [34]. In [40], streamer branching was simulated by artificially decreasing the importance of photo-ionization and increasing the streamer diameter. On the other hand, the density fluctuations due to the discrete particle nature of electrons as modeled, e.g., in $[3,4,5,6,7,8]$ might introduce a destabilizing effect. The search is ongoing.

\subsection{Planar streamer ionization fronts and their pulled dynamics}

We now turn to analytical results on streamer ionization fronts that can shed further light on the simulations. We first recall essential properties of planar fronts and in the next subsection their stability properties.

The non-ionized state with high electric field ahead of a streamer ionization front is linearly unstable: electrons escaping from the front will form their own avalanches. Within the minimal streamer model, electrons reach the non-ionized region by diffusion. The diffusive leading edge of the front determines the velocity and pulls the front along. The oldest and most studied equation forming pulled fronts is the Fisher-KPP-equation, but streamer fronts are pulled as well [27, 28, 63].

If the initial electron density is very extended ahead of the front, this extended leading edge will grow everywhere and pull the front along with high velocity. (We remark that in practice the particle nature of the electrons will truncate such a leading edge at low densities.) But if the initial electron density of a negative ionization front in the minimal model (1)-(3) decays sufficiently rapidly into the unstable state,

$$
\lim _{z \rightarrow \infty} \sigma(x, y, z, t=0) e^{\lambda z}=0 \text { for all } \lambda<\Lambda^{*}=\sqrt{\frac{\left|E^{+}\right| \alpha\left(E^{+}\right)}{D}}
$$

the front velocity in a field $E^{+}$will converge to $[27,28,63]$

$$
v^{*}\left(E^{+}\right)=\left|E^{+}\right|+2 \sqrt{D\left|E^{+}\right| \alpha\left(E^{+}\right)} .
$$

The spatial profiles and other properties of these fronts are summarized and illustrated in section 2 of [64] for varying $E^{+}$and $D$.

Also for positive ionization fronts, a unique asymptotic velocity is selected in the minimal model by a sufficiently rapid decay of the electron density. However, the dynamics of negative fronts for $\left|E^{+}\right|=\mathcal{O}(1)$ and $D=0.1$ is dominated by the drift term $\left|E^{+}\right|$, and the limit $D \rightarrow 0$ is smooth for all relevant quantities. In contrast, for positive fronts, the velocity vanishes as the singular perturbation $D$ vanishes, while the ionization density behind the front diverges [28]. For the physically relevant $D=0.1$ the resulting front velocity for positive fronts is so low that other physical effects should be included, either photo-ionization or background ionization or at least ion mobility.

Planar positive ionization fronts with photo-ionization probably keep accelerating while a curved front does not. This observation again is related to the linear instability of the penetrated state. While this unstable state extends to infinity ahead of a planar front because the electric field ahead of a planar front is constant, the instability occurs 
only in a finite spatial range ahead of a curved front. This is because the electric field ahead of a curved front decays on a length scale determined by the radius of curvature. This decay of the field limits the spatial region where the non-ionized state is linearly unstable.

The pulled nature of the diffusive leading edge also forms a challenge the numerical solution of the density model [59] and of particle models [3, 6]. Again the problem is particularly severe for planar fronts. For curved fronts, the pulled region becomes an intermediate region where the electric field does not vary much yet, while the stability of the non-ionized state is restored where the electric field drops below the break-down value.

\subsection{Stability of negative planar ionization fronts with $D=0$ or $D \neq 0$}

The stability of transversal perturbations of planar negative ionization fronts in the minimal model (1)-(3) was analyzed in [65, 64], using the ansatz

$$
\begin{aligned}
(\sigma, \rho, E)(x, z, t)= & \left(\sigma_{0}, \rho_{0}, E_{0}\right)(\xi)+\left(\tilde{\sigma}_{k}, \tilde{\rho}_{k}, \tilde{E}_{k}\right)(\xi) e^{i k x+s t}+\ldots, \\
& \xi=z-v^{*} t .
\end{aligned}
$$

Here $\left(\sigma_{0}, \rho_{0}, E_{0}\right)(\xi)$ denotes a planar front that propagates uniformly with the pulled front velocity $v^{*}\left(E_{\infty}\right)$, given in Eq. (5), into a non-ionized region with constant electric field $E_{\infty}$. $\left(\tilde{\sigma}_{k}, \tilde{\rho}_{k}, \tilde{E}_{k}\right)(\xi)$ is a linear perturbation with wave number $k$. Here $E_{\infty}$ is the field far ahead of the front. The field $E^{+}$just ahead of the front is identical to $E_{\infty}$ for the planar front $\left(\sigma_{0}, \rho_{0}, E_{0}\right)(\xi)$, but $E^{+}$deviates from $E_{\infty}$ when the front is perturbed.

Because the state $\sigma=0$ is linearly unstable, the electron density has to obey the decay constraint (4) to define a unique asymptotic front velocity $v^{*}$ and also to define a unique dispersion relation $s(k)$.

For the case $D=0$, the calculation of the dispersion relation was briefly summarized in [53] and fully elaborated in [65]. It is

$$
s(k)=\left\{\begin{array}{ll}
\left|E_{\infty}\right| k & \text { for } k \ll \alpha\left(E_{\infty}\right) / 2 \\
\left|E_{\infty}\right| \alpha\left(E_{\infty}\right) / 2 & \text { for } k \gg \alpha\left(E_{\infty}\right) / 2
\end{array} .\right.
$$

Hence planar ionization fronts are dynamically unstable against perturbations of any wave length, and the growth rate of perturbations saturates to a constant positive value for sufficiently large wave number $k$. This means that perturbations composed of Fourier modes with large wave numbers $k$ will grow as $e^{s(\infty) t}$ without changing spectral composition.

As the instability of arbitrarily small wave lengths appears unphysical, in [64] the transverse instability for streamer fronts with $D \neq 0$ was analysed. Indeed when diffusion is included, the band of unstable wave lengths becomes finite. Here is a summary of method and results.

Along the dispersion curve $s(k)$, the linearised system has a solution that is bounded for all $z$. In other words, the unstable manifold for $z \rightarrow-\infty$ and the stable manifold for $z \rightarrow \infty$ have a non-trivial intersection. The intersection can be determined with 
the Evans function, an analytic function whose zeros correspond to the eigenvalues of a spectral problem, see $[66,67,68,69,70]$. Hence the dispersion curve $s(k)$ can be found as a curve of zeros of an Evans function with the far field $E_{\infty}$ and the electron diffusion $D$ as parameters. In case of a pulled front, the stable manifold has to be defined carefully, as the temporal stability of the asymptotic state of the pulled front at $+\infty$ is conditional on the spatial decay of the perturbation (4). To build this decay condition into the definition of the stable manifold, the stable manifold and Evans function are defined on a weighted space.

Using exterior algebra, the Evans function can be evaluated numerically and dispersion curves are obtained. Numerical results on the dependence of the dispersion relation on $E_{\infty}$ and $D$ are included in Fig. 7. Furthermore, the asymptotic limits for large and small wave lengths can be determined analytically:

$$
s(k)=\left\{\begin{aligned}
k E_{\infty} \frac{d v^{*}}{d E_{\infty}}, & k \ll 1 \\
-D k^{2}, & k \gg 1 .
\end{aligned}\right.
$$

In spite of the fact that $D=0$ is a singular perturbation, the asymptotic limit for large wave numbers converges to the expression (7) for $D=0$.

A (heuristic) analysis for $s(k)$ was given in [71, 72]. The asymptotes (8) confirm these results for $k \gg 1$, and correct them for $k \ll 1$, see also [73]. On the other hand, the analytical asymptote (8) for $k \ll 1$ fits the numerical results for the dispersion relation well, while the asymptote for $k \gg 1$ parameterizes the dispersion relation $s(k)$ deep in its negative range, but not close to the band of wave numbers of positive growth rate [64]. According to a physically motivated empirical fit to the numerical data shown in Fig. 7 , the wave number with maximal growth rate is approximately $\Lambda^{*} / 16$, and the growth rate $s(k)$ is positive for $k \leq c \Lambda^{*}, c \approx 1 / 4$, with $\Lambda^{*}$ defined in (4).

\section{Derivation of a moving boundary approximation for negative streamer fronts}

\subsection{Basic observations}

Inspecting the numerical results for negative streamers without photo-ionization, as displayed e.g. in Fig. 6, we observe some important features.

1. The active space charge layer is very thin. Its thickness is much smaller than its radius of curvature. This amounts to a separation of scales, with the inner scale being defined by the thickness, the outer scale being given by the radius.

2. Inside the streamer the electric field is very small.

3. For sufficiently large field the streamer moves with a velocity $\mathbf{v}$ proportional to the electric field at the tip.

4. Furthermore the region outside the streamer contains no charges, and far from the streamer the electric field becomes constant. 
These observations suggest to model the dynamics of the streamer on the outer scale as a moving boundary problem. A sketch is shown in Fig. 8, and points 1-4 lead to the following set of basic equations:

$$
\begin{array}{ll}
\text { outer region: } & \nabla^{2} \varphi=0, \\
& \mathbf{E}(\mathbf{r}, t) \rightarrow E_{\infty} \hat{\mathbf{x}}, \\
& \varphi=0, \\
\text { inner region: } & \mathbf{v}(\mathbf{r}, t) \propto \mathbf{E}^{+}(\mathbf{r}, t),
\end{array}
$$

where $\mathbf{E}^{+}$is the limit defined by approaching the boundary from the outer region, and $\hat{\mathbf{x}}$ is the unit vector in $x$ direction. The limit taken from the interior will be indicated by a superscript (-). Taking the potential to be continuous at the interface,

$$
\varphi^{+}-\varphi^{-}=0,
$$

this model was first proposed by Lozansky and Firsov and described in a Russian textbook; a short account in English can be found in [74]; it contains the approximation and finds parabolas to be uniformly translating solutions in two dimensional space. Independently the model was suggested in [75] where it was placed in the context of other pattern forming problems with moving boundaries like dendritic growth of solidification fronts or viscous fingers in two-fluid flow in Hele-Shaw cells; this relation was already suggested in $[27,28]$. In fact, with proper reinterpretation of the quantities the model coincides with the (non-regularized) model for Hele-Shaw flow.

It should be clear from Subsect. 3.3 that the moving boundary model can be valid only for negative streamers. As pointed out there, the front for nonvanishing diffusion, $D \neq 0$, is pulled, and its dynamics is determined in some region ahead of the front. A priori this is incompatible with (12). It thus is not surprising that an attempt to formally derive a moving boundary model from the minimal streamer model with $D \neq 0$ failed [76]. The solvability (or Fredholm) analysis leads to divergencies. However, as also pointed out in Subsect. 3.3, for a negative front the limit $D \rightarrow 0$ is smooth, and furthermore $D$ is small experimentally. Thus eliminating the pulled nature of the front by taking $D=0$ is a fair approximation for negative fronts, but is invalid for positive fronts where the limit $D \rightarrow 0$ is singular.

\subsection{Boundary condition at the interface}

As is well known, the model with the boundary condition (b.c.) (13) is mathematically ill posed, (see e.g. Ref. [77] and references therein). Depending on the initial condition the moving boundary may develop singularities within arbitrarily short time. The b.c. (13) completely ignores the physics of the space charge layer on the inner scale. In deriving a proper b.c., some integrated property of the layer has to be taken into account. Since by definition $\varphi^{+}-\varphi^{-}$is the change of the potential over the space charge layer, the simplest approach to determine this difference consists in integrating over the analytically known expression for a planar front. This yields

$$
\varphi^{+}-\varphi^{-}=F\left(E^{+}\right), \quad E^{+}=\hat{\mathbf{n}} \cdot \nabla \varphi^{+}
$$




$$
\begin{aligned}
& F(E)=\int_{0}^{E} d z \frac{E-z}{\bar{\rho}(z, E)}, \\
& \bar{\rho}(z, E)=\int_{z}^{E} d y \alpha(y),
\end{aligned}
$$

where $\alpha$ is the Townsend ionization coefficient introduced in the minimal streamer model, $\bar{\rho}$ is the ion density expressed as a function of the local electric field and $\hat{\mathbf{n}}$ is the unit vector normal to the interface and oriented toward the non-ionized region. (For convenience of notation, the field $E^{+}=\hat{\mathbf{n}} \cdot \nabla \varphi^{+}$is defined with reversed sign in this section.) For large field, $F(E)$ becomes proportional to $E$ and (14) reduces to

$$
\varphi^{+}-\varphi^{-}=\ell E^{+}
$$

where the length $\ell$ is of the order of the thickness of the charge layer. It is this b.c. which will be used in the sequel. We note that in the context of dendritic growth, Eq. (17) is known as 'kinetic undercooling' condition. With the b.c. (17), the moving boundary model for perturbations of a planar front yields a dispersion relation which for small or large $k$ coincides with the result (6) derived from the minimal streamer model with $D=0$. Furthermore, numerical solutions of the minimal streamer model including diffusion show (17) to be a good approximation in the leading active part of the streamer front [78].

Curvature corrections to the b.c. (17) have been calculated to first order via a Fredholm analysis of the minimal streamer model with $D=0$ in 2 dimensions. The result confirmed the b.c (17) and added a curvature correction as well as a prediction on the electric field behind a uniformly translating weakly curved front [79]:

$$
\begin{aligned}
\varphi^{+}-\varphi^{-} & =F\left(E^{+}\right)+Q_{1}\left(E^{+}\right) \kappa, \quad Q_{1}(x) \stackrel{|x| \gg 1}{\longrightarrow}-\frac{\pi^{2}}{6} x \\
\hat{\mathbf{n}} \cdot \nabla \varphi^{-} & =\frac{E^{+2} \kappa}{\bar{\rho}\left(0, E^{+}\right)} \\
\mathbf{v} & =\nabla \varphi^{+},
\end{aligned}
$$

where $\kappa$ is the local curvature of the front. The full analytical expression of the nonlinear function $Q_{1}(x)$ can be found in Ref. [79]. The approximation was successfully tested on numerical solutions of the minimal streamer model in [80]; a generalization of the interesting result on the field $\hat{\mathbf{n}} \cdot \nabla \varphi^{-}$inside the propagating streamer will be presented in $[26]$.

\section{Analysis of the moving boundary model in two dimensions}

\subsection{Analytical and numerical results}

We here summarize the results of $[81,82,83,84]$.

Rescaling field, space and time, the model in two-dimensional space $(x, y)$ can be reduced to the following form that is also illustrated in Fig. 8. Outside the streamer (scaled to the area $\pi$ ), in the region $\mathcal{D}_{c}$, the electric potential $\varphi$ satisfies

$$
\Delta \varphi=0
$$


with interfacial conditions:

$$
v_{n}=\frac{\partial \varphi}{\partial n}, \quad \varphi=\epsilon \mathbf{n} \cdot \nabla \varphi
$$

where $v_{n}$ is the interfacial normal speed, and $\epsilon=\ell / R$ is the ratio of the inner to the outer scale. A uniform electric field is approached far from the streamer, implying

$$
\mathbf{E}=-\nabla \varphi \rightarrow-\hat{\mathbf{x}}+o(1 /|\mathbf{x}|)
$$

where $\hat{\mathbf{x}}$ is the unit vector in the $x$-direction. Eqs. (21)-(23) define our model.

We introduce the time dependent conformal map $z=x+i y=f(\omega, t)$ that maps the unit disk $\mathcal{U}_{\omega}$ into $\mathcal{D}_{c}$ and a corresponding complex potential $\Phi(\omega, t)$, with $\varphi=\operatorname{Re} \Phi$.

$$
z=f(\omega, t)=\frac{a_{-1}(t)}{\omega}+\hat{f}(\omega, t), \quad \Phi(\omega, t)=\frac{a_{-1}(t)}{\omega}+\hat{\Phi}(\omega, t) . \quad a_{-1}(t)>0 .
$$

Both $\hat{f}(\omega, t)$ and $\hat{\Phi}(\omega, t)$ are analytic for $\omega \in \mathcal{U}_{\omega}$. The interfacial boundary conditions (22) imply

$$
\begin{aligned}
& \operatorname{Re}\left[\frac{\partial_{t} f}{\omega \partial_{\omega} f}\right]=\operatorname{Re}\left[\frac{\omega \partial_{\omega} \Phi}{\left|\partial_{\omega} f\right|^{2}}\right], \quad \text { for }|\omega|=1 \\
& \left|\partial_{\omega} f\right| \operatorname{Re} \Phi=-\epsilon \operatorname{Re}\left[\omega \partial_{\omega} \Phi\right], \quad \text { for }|\omega|=1 .
\end{aligned}
$$

For $\epsilon>0$, the only known completely smooth exact solution is the unit circle moving with constant velocity $2 /(1+\epsilon)$ in $x$-direction. Formulated in terms of $f$ and $\Phi$, this solution reads

$$
\begin{aligned}
f^{(0)}(\omega, t) & =\frac{1}{\omega}+\frac{2 t}{1+\epsilon}, \\
\Phi^{(0)}(\omega, t) & =\frac{1}{\omega}-\frac{1-\epsilon}{1+\epsilon} \omega .
\end{aligned}
$$

Relaxing somewhat the smoothness constraint, other uniformly translating solutions can be constructed [85].

We here consider linear perturbations of the circle, making the ansatz

$$
\begin{aligned}
& f(\omega, t)=f^{(0)}(\omega, t)+\eta \beta(\omega, t) \\
& \Phi(\omega, t)=\Phi^{(0)}(\omega, t)+\eta \frac{2}{1+\epsilon} \chi(\omega, t),
\end{aligned}
$$

where $\beta(\omega, t)$ and $\chi(\omega, t)$ are analytic in $\mathcal{U}_{\omega}$ and $\eta$ is a small parameter. Substituting (29) into equations (25), (26), the leading order equation for small $\eta$, on elimination of $\chi$ leads to

$$
\mathcal{L}_{\epsilon} \beta=0 \text { where } \mathcal{L}_{\epsilon}=\frac{\epsilon}{2} \partial_{\omega}\left(\omega^{2}-1\right) \omega \partial_{\omega}+\epsilon \partial_{\omega} \omega \partial_{\tau}+\partial_{\tau}-\partial_{\omega}
$$

where $\tau=\frac{2}{1+\epsilon} t$ is re-scaled time. When $\epsilon=1$, the solution is known analytically $[81,82]$ :

$$
\beta(\omega, \tau)=\frac{1}{\omega^{2}} \int_{0}^{\omega} \omega^{\prime} G\left(\frac{\omega^{\prime}+T}{1+\omega^{\prime} T}\right) d \omega^{\prime}
$$


where the function $G(\omega)=\left(2+\omega \partial_{\omega}\right) \beta(\omega, 0)$ and $T(\tau)=\tanh \frac{\tau}{2}$. The properties of these solutions are discussed and visualized in detail in $[81,82]$. Here we in particular note that the essential time dependence of $\beta(\omega, \tau)$ is contained in the transformation

$$
\zeta=\frac{\omega+T(\tau)}{1+\omega T(\tau)}
$$

$\zeta(\omega, T), 0 \leq T \leq 1$, defines a one-parameter family of automorphisms of the unit disk, with fixed points $\omega= \pm 1$. The point $\omega=1$ is stable, whereas $\omega=-1$ is unstable in the following sense: as $\tau \rightarrow \infty$, i.e. $T \rightarrow 1$, all the complex $\omega$-plane, except for $\omega=-1$, is mapped into a neighborhood of $\zeta=+1$. This results in an advective dynamics. Any perturbation not centered precisely at $\omega=1$ is advected towards $\omega=-1$, where it vanishes asymptotically. As $\tau \rightarrow \infty$, only a shift of the circle is left:

$$
\lim _{\tau \rightarrow \infty} \beta(\omega, \tau)=\frac{G(1)}{2} .
$$

For $\epsilon \neq 1$ the same automorphism (32) describes the advection of small disturbances from the front to the back of the bubble. Outside an arbitrarily small, but fixed neighbourhood of $\omega=-1$, the behaviour of $\beta(\omega, \tau)$ for $\tau \rightarrow \infty$ on the circle $\omega=e^{-\alpha}$ is given by

$$
\beta(\omega, \tau)=\sum_{j=0}^{\infty} e^{\lambda_{j} t} \beta_{j}(\omega) .
$$

where $\left(\lambda_{j}, \beta_{j}\right)$ are the eigenvalues and the corresponding eigenfunctions which have a singularity at $\omega=-1$. The spectrum has been proven to be discrete, and except for the trivial translation mode $\lambda_{0}=0, \beta_{0}(\omega)=1$, all eigenvalues are in the left half complex plane. Indeed, analytical arguments and numerical work suggest that all $\lambda_{j}(\epsilon), j \geq 1$, are negative, converging to $\lambda_{j}(\epsilon)=0$ for $\epsilon \rightarrow 0$. Fig. 9 shows the behaviour for the lowest eigenvalues.

Near the back of the circle, in a region of size $(1+\omega) e^{\tau}=O(1)$, the relaxation is not given by Eq. (34), but takes the form

$$
\tilde{\beta}(\zeta, T(\tau))=e^{-\tau / \epsilon} \hat{\beta}(\zeta, \tau)+\sum_{k=0}^{\infty} \hat{\gamma}_{k}(\zeta) e^{-k \tau}(\zeta, \tau)
$$

Here

$$
\tilde{\beta}(\zeta(\omega, T(\tau)), T(\tau))=\beta(\omega, \tau),
$$

is used to transform from variables $(\omega, \tau)$ to variables $(\zeta, T)$.

The numerical results for linear evolution support the analytical results mentioned above. Small initial perturbations increase as long as they are in the front half of the circle, but are convected to the back where they decay. For the nonlinear evolution numerical results indicate that the circle is the asymptotic attractor for small perturbations, but larger perturbations may lead to branching. This is illustrated in Fig. 10 with the initial condition $\beta(\omega, 0)=-0.03 \omega^{5}$. Panel (a) shows snapshots of the interface in physical space $z=x+i y=f(\omega, t)$, with $\epsilon=\frac{1}{10}$, as resulting from the nonlinear evolution. For comparison panel (b) shows the linearized evolution. Snapshots 
are taken at times $t=0.05 n$, where $n=0,1, \ldots, 12$ in panels (a) and (b). A qualitative effect of the nonlinearity is observed for $t>0.1$. Whereas the linear approximation develops shoulders connected by some flat part of the interface, the nonlinear evolution results in two branches separated by a valley. Since the bottom of the valley moves slower than the tips of the branches, the valley is likely to evolve into a deep fjord. For more general initial shapes, the interfaces are not guaranteed to be smooth globally in time with the present regularization by the "kinetic undercooling" boundary condition.

\subsection{Consequences for the evolution of streamers}

Clearly, a circle is not a faithful representation of a physical streamer, even in an essentially two-dimensional geometry. The moving boundary model is extracted from the physics of the front part of the streamer and cannot describe the back part where the ionized region in the laboratory extends to some electrode or in nature to a thundercloud or the ionosphere. Therefore, the relaxation at the back of the circle, as described above, is of mathematical interest, but is not physical.

Nevertheless, we believe that the results of the moving boundary approximation are physically meaningful since both in streamers and in the model, it is the front part that dominates the dynamics. This view is supported by the results of the next section, illustrating the close similarity of special solutions of the density model with classical Saffman-Taylor fingers [86]. Accepting this view, we can state that negative streamer fronts are convectively stabilized and branching occurs only for sufficiently large perturbations. This dynamics occurs similarly in viscous fingering with a small surface tension regularization (see for instance [54]).

\section{Interacting streamers and the relation to the Saffman-Taylor problem}

\subsection{Streamer groups and the selected Saffman-Taylor finger}

The moving boundary solutions above show that indeed the regularization condition (17) is sufficient to stabilize a curved moving streamer front by convection. But the approximation that a sharp boundary moves with the electron drift velocity becomes questionable at the sides of the moving streamer and invalid at the back. We now present a case where the approximation for the front velocity (12) is needed essentially only in its range of validity. This is the case for a growing finger in a laterally constrained space.

In the case of the classical Saffman-Taylor finger in two-fluid flow, the lateral constraint is given by a solid wall at the side of the Hele-Shaw cell. But an equivalent wall cannot be constructed for an electrodynamic problem from any type of matter: the lateral boundary condition in the Saffman-Taylor fluid problem requires that the normal component of the pressure gradient $\nabla p$ vanishes (where $p$ in the hydrodynamic problem corresponds to $\varphi$ in streamers), but a conducting medium only can support the tangential component of the electric field $\mathbf{E}=-\nabla \varphi$ to vanish. However, if a group of identical streamers propagates next to each other, on the symmetry plane 
between two streamers the normal component of the electric field vanishes. Therefore the mathematical setting of the Saffman-Taylor problem in a channel of lateral width $L$ is equivalent to the streamer moving boundary problem in two dimensions when the distance between the centers of identical streamers is $L$. The only difference lies in the different regularization conditions on the boundary.

Fig. 11 shows a plot from [87]. It compares the solutions of the minimal streamer density model for a periodic streamer group in two dimensions with the selected solution of the Saffman-Taylor problem; this solution is parameterized in the $(x, y)$ plane by

$$
x=\frac{L(1-\lambda)}{2 \pi} \ln \cos ^{2} \frac{\pi y}{\lambda L}+\frac{\left|E_{\infty}\right|}{\lambda} t
$$

with $\lambda=1 / 2$. Here $E_{\infty}$ is the electric field far ahead of the streamer group. As also other results in [87] show, the specific Saffman-Taylor finger (37) with $\lambda=1 / 2$ parameterizes the streamer fingers in two dimensions very well.

Without regularization, Saffman and Taylor found that a uniformly translating finger in a Hele-Shaw cell can have an arbitrary width $\lambda$ within the channel [86], and the same is, of course, true for the streamer fingers in a group, as the mathematical problem is the same. But surface tension regularization (see $[88,89,90]$ and references therein) selects a unique finger whose width is half the channel width, $\lambda=1 / 2$. But the same solution is also selected by the "kinetic undercooling" regularization [91]. The fact that the numerical solutions of the PDE streamer model approach this solution therefore supports the validity of our moving boundary approximation.

We remark that a similar selection takes place for a bubble in Hele-Shaw flow or for the compact two-dimensional "streamer bubbles" discussed in the last section. Without regularization, all uniformly translating ellipses with main axes oriented parallel and perpendicular to the field or flow are solutions. When surface tension or kinetic undercooling is introduced, only uniformly translating circles are solutions [92, 93, 81]. In the limit of small regularization parameter, they enhance the electric field by a factor of 2 , just like the selected Saffman-Taylor fingers.

\subsection{Field enhancement at the streamer tip}

The fact that the electric field at the tip of the streamers in the two-dimensional group is $2 E_{\infty}$, i.e., the field is enhanced at the tip by a factor of 2 with respect to the background field, is a physically most interesting result. As said above, the same enhancement factor is found at the front of the two-dimensional streamer "bubbles" treated in the last section. (For single streamers, this enhancement factor is much higher, cf. Figs. 6 and 7 and [26].) As the field is enhanced by a factor of 3 at the front part of an ideally conducting sphere in a homogeneous field in three dimensions, it can be conjectured that the field enhancement at the tips of a three-dimensional streamer group is 3 as well. This is subject of further study. 


\section{Conclusion and outlook}

Streamers are ubiquitous in nature and technology. Their understanding involves many scales from the microscopic scale of collisions of electrons with neutral molecules to a hierarchy of macroscopic scales ranging from thin space charge layers within each streamer finger up to the streamer tree with possibly thousands of branches.

After a short introduction to observations, experiments and simulations, the article emphasizes on density or PDE approximations for negative streamer fronts and on moving boundary approximations, and on the stability of these fronts and boundaries.

The analysis of the ionization fronts is non-standard due to their pulled dynamics. This pulled dynamics can be removed by completely neglecting the small electron diffusion constant $D$. For $D=0$, planar ionization fronts are linearly unstable against perturbations of any wave length; a finite band of unstable wave lengths exists for $D>0$. Nevertheless, curved fronts in a moving boundary model with $D=0$ and without curvature correction turn out to be linearly stable. In fact, while perturbations are growing at the front part of the front, they are convected away. Therefore a finite perturbation is necessary to destabilize the streamer tip and make it branch.

The analysis of a moving compact streamer allowed us to proceed far in an analytical stability analysis. In doing so, we have to take some unphysical boundary conditions at the back of the object into account, but as the dynamics is determined at the propagating tip, this does not matter.

For streamer fingers in periodic groups of identical streamers in two dimensions, we found that the classical Saffman-Taylor finger solution fits numerical PDE solutions very well, though the regularization conditions of the respective moving boundary models differ - the mathematical reason for this coincidence is that the "kinetic undercooling" regularization selects the same solution as regularization by surface tension.

The main analytical results reviewed here concern negative streamers in twodimensional space. Clearly future work must concentrate on the more physical problem of streamers in three dimensions, and must address the practically more important case of positive streamers. The phenomena observed in positive and negative streamers are similar, both in experiment and simulations. But the underlying mathematics is quite different.

Both in experiment and in nature, generally many streamers are observed simultaneously. The results on Saffman-Taylor streamers reviewed here are only a first step in understanding the mutual interaction of streamers. Clearly much work needs to be done before the full streamer tree is understood.

Acknowledgement: U.E. and V.R. acknowledge support by the Netherlands' IOP-EMVT under contract number 062126B. 


\section{References}

[1] S. Nijdam, F.M.J.H. van de Wetering, R. Blanc, E.M. van Veldhuizen, U. Ebert, Probing photoionization: Experiments on positive streamers in pure gases and mixtures, J. Phys. D: Appl. Phys. 43 (2010) 145204.

[2] G. Wormeester, S. Pancheshnyi, A. Luque, S. Nijdam, U. Ebert, Probing photo-ionization: Simulations of positive streamers in varying $\mathrm{N}_{2}: \mathrm{O}_{2}$-mixtures, J. Phys. D: Appl. Phys. [in print - D/365202/PAP/255631, page proofs are done], arXiv:1008.3309v2.

[3] C. Li, W.J.M. Brok, U. Ebert, J.J.A.M. van der Mullen, Deviations from the local field approximation in negative streamer heads, J. Appl. Phys. 101 (2007) 123305.

[4] O. Chanrion, T. Neubert, A PIC-MCC code for simulation of streamer propagation in air, J. Comput. Phys. 227 (2008) 7222.

[5] C. Li, U. Ebert, W. Hundsdorfer, 3D hybrid computations for streamer discharges and production of run-away electrons, J. Phys. D: Appl. Phys. 42 (2009) 202003.

[6] C. Li, U. Ebert, W. Hundsdorfer, Spatially hybrid computations for streamer discharges with generic features of pulled fronts: I. Planar fronts, J. Comput. Phys. 229 (2010) 200.

[7] O. Chanrion, T. Neubert, Production of runaway electrons by negative streamer discharges, J. Geophys. Res. 115 (2010) A00E32.

[8] C. Li, U. Ebert, W. Hundsdorfer, Spatially hybrid computations for streamer discharges: II. Fully $3 D$ simulations, submitted to J. Comput. Phys.

[9] U. Ebert, C. Montijn, T.M.P. Briels, W. Hundsdorfer, B. Meulenbroek, A. Rocco, E.M. van Veldhuizen, The multiscale nature of streamers, Plasma Sources Sci. Technol. 15 (2006) S118.

[10] P. Blom, C. Smit, R.H. Lemmens, E.J. van Heesch, Combined optical and electrical measurements on pulsed corona discharges, page 609 in Gaseous Dielectrics VII, eds.: L.G. Christophorou and D. James, Plenum Press, New York, 1994.

[11] P.P.M. Blom, High-power pulsed corona, Ph.D. thesis at Eindhoven Univ. Techn., supervised by P.C.T. van der Laan, F.J. de Hoog and E.J.M. van Heesch, http://alexandria.tue.nl/extra1/PRF14A/9702338.pdf.

[12] S. Pancheshnyi, M. Nudnova, A. Starikovskii, Development of a cathode-directed streamer discharge in air at different pressures: Experiment and comparison with direct numerical simulation, Phys. Rev. E 71 (2005) 016407.

[13] G.J.J. Winands, Z. Liu, A.J.M. Pemen, E.J.M. van Heesch, K. Yan, Analysis of streamer properties in air as function of pulse and reactor parameters by ICCD photography, J. Phys. D: Appl. Phys. 41 (2008) 234001.

[14] T.M.P. Briels, E.M. van Veldhuizen, U. Ebert, Time resolved measurements of streamer inception in air, IEEE Trans. Plasma Sci. 36 (2008) 908.

[15] T.M.P. Briels, J. Kos, G.J.J. Winands, E.M. van Veldhuizen, U. Ebert, Positive and negative streamers in ambient air: measuring diameter, velocity and dissipated energy, J. Phys. D: Appl. Phys. 41 (2008) 234004.

[16] T.M.P. Briels, E.M. van Veldhuizen, U. Ebert, Positive streamers in air and nitrogen of varying density: experiments on similarity laws, J. Phys. D: Appl. Phys. 41 (2008) 234008.

[17] M.M. Nudnova, A.Yu. Starikovskii, Streamer head structure: role of ionization and photoionization, J. Phys. D: Appl. Phys. 41 (2008) 234003.

[18] S. Nijdam, J.S. Moerman, T.M.P. Briels, E.M. van Veldhuizen, U. Ebert, Stereo-photography of streamers in air, Appl. Phys. Lett. 92 (2008) 101502.

[19] S. Nijdam, C.G.C. Geurts, E.M. van Veldhuizen, U. Ebert, Reconnection and merging of positive streamers in air, J. Phys. D: Appl. Phys. 42 (2009) 045201.

[20] U. Ebert, S. Nijdam, C. Li, A. Luque, T.M.P. Briels, E.M. van Veldhuizen, Review of recent results on streamer discharges and their relevance for sprites and lightning, J. Geophys. Res. 115 (2010) A00E43.

[21] U. Ebert, D.D. Sentman, Streamers, sprites, leaders, lightning: from micro- to macroscales, review 
and editorial introduction to a cluster issue on "Streamers, Sprites and Lightning", J. Phys. D: Appl. Phys. 41 (2008) 230301.

[22] R.D. White, K.F. Ness, R.E. Robson, Development of swarm transport theory in radio-frequency electric and crossed electric and magnetic fields, Appl. Surf. Sci. 192 (2002) 26.

[23] R.D. White, R.E. Robson, S. Dujko, P. Nicoletopoulos, B. Li, Recent advances in the application of Boltzmann equation and fluid equation methods to charged particle transport in non-equilibrium plasmas, J. Phys. D: Appl. Phys. 42 (2009) 194001.

[24] S. Dujko, R.D. White, Z.Lj. Petrovic, R.E. Robson, Benchmark calculations of nonconservative charged-particle swarms in dc electric and magnetic fields crossed at arbitrary angles, Phys. Rev. E 81 (2010) 046403.

[25] R.D. White, S. Dujko, R.E. Robson, Z.Lj. Petrovic, R.P. McEacran, Non-equilibrium transport of positron and electron swarms in gases and liquids, Plasma Sources Sci. Technol. 19 (2010) 034001.

[26] V. Ratushnaya, A. Luque, U. Ebert, Electrodynamic characterization of long positive streamers in air, in preparation for J. Phys. D: Appl. Phys.

[27] U. Ebert, W. van Saarloos, and C. Caroli, Streamer Propagation as a Pattern Formation Problem: Planar Fronts, Phys. Rev. Lett. 77 (1996) 4178.

[28] U. Ebert, W. van Saarloos, and C. Caroli, Propagation and Structure of Planar Streamer Fronts, Phys. Rev. E 55 (1997) 1530.

[29] S.K. Dhali, P.F. Williams, Numerical simulation of streamer propagation in nitrogen at atmospheric pressure, Phys. Rev. A 31 (1985) 1219.

[30] S.K. Dhali, P.F. Williams, Two-dimensional studies of streamers in gases, J. Appl. Phys. 62 (1987) 4696.

[31] C. Wu, E.E. Kunhardt, Formation and propagation of streamers in $N_{2}$ and $N_{2}-S F_{6}$ mixtures, Phys. Rev. A 37 (1988) 4396.

[32] P.A. Vitello, B.M. Penetrante, J.N. Bardsley, Simulation of negative-streamer dynamics in nitrogen, Phys. Rev. E 49 (1994) 5574.

[33] G.D. Moss, V.P. Pasko, N. Liu, G. Veronis, Monte Carlo model for analysis of thermal runaway electrons in streamer tips in transient luminous events and streamer zones of lightning leaders, J. Geophys. Res. 111 (2006) A02307.

[34] A. Luque, U. Ebert, C. Montijn, W. Hundsdorfer, Photoionisation in negative streamers: fast computations and two propagation modes, Appl. Phys. Lett. 90 (2007) 081501.

[35] N.Y. Babaeva, G.V. Naidis, Two-dimensional modelling of positive streamer dynamics in nonuniform electric fields in air, J. Phys. D: Appl. Phys. 29 (1996) 2423.

[36] A.A. Kulikovsky, Positive streamer between parallel plate electrodes in atmospheric pressure air, J. Phys. D: Appl. Phys. 30 (1997) 441.

[37] A.A. Kulikovsky, The role of photoionization in positive streamer dynamics, J. Phys. D: Appl. Phys. 33 (2000) 1514.

[38] S.V. Pancheshnyi, S.M. Starikovskaia, A.Y. Starikovskii, Role of photoionization processes in propagation of cathode-directed streamer, J. Phys. D: Appl. Phys. 34 (2001) 105.

[39] S.V. Pancheshnyi, A.Y. Starikovskii, Two-dimensional numerical modelling of the cathode-directed streamer development in a long gap at high voltage, J. Phys. D: Appl. Phys. 36 (2003) 2683.

[40] N. Liu, V.P. Pasko, Effects of photoionization on propagation and branching of positive and negative streamers in sprites, J. Geophys. Res. (Space Phys) 109 (2004) 4301.

[41] N. Liu, V.P. Pasko, Effects of photoionization on similarity properties of streamers at various pressures in air, J. Phys. D: Appl. Phys. 39 (2006) 327.

[42] O. Eichwald, O. Ducasse, D. Dubois, A. Abahazem, N. Merbahi, M. Benhenni, M. Yousfi, Experimental analysis and modelling of positive streamer in air: towards an estimation of $O$ and $N$ radical production, J. Phys. D 41 (2008) 234002.

[43] A. Luque, V. Ratushnaya, U. Ebert, Positive and negative streamers in ambient air: modelling evolution and velocities, J. Phys. D: Appl. Phys. 41 (2008) 234005. 
[44] A. Luque, U. Ebert, Emergence of sprite streamers from screening-ionization waves in the lower ionosphere, Nature Geoscience 2 (2009) 757.

[45] N. Liu, Model of sprite luminous trail caused by increasing streamer current, Geophys. Res. Lett. 37 (2010) 4102.

[46] A. Luque, U. Ebert, Sprites in varying air density: charge conservation, glowing negative trails and changing velocity, Geophys. Res. Lett. 37 (2010) L06806.

[47] D. Dubrovin, S. Nijdam, E.M. van Veldhuizen, U. Ebert, Y. Yair, C. Price, Sprite discharges on Venus and Jupiter-like planets: A laboratory investigation, J. Geophys. Res - Space Phys. 115 (2010) A00E34.

[48] S. Pancheshnyi, Role of electronegative gas admixtures in streamer start, propagation and branching phenomena, Plasma Sour. Sci. Technol. 14 (2005) 645.

[49] S.A. Cummer, J. Li, W.A. Lyons, T.E. Nelson, E.A. Gerken, Submillisecond imaging of sprite development and structure, Geophys. Res. Lett. 33 (2006) 4104.

[50] H.C. Steenbaek-Nielsen, M.G. McHarg, High time-resolution sprite imaging: observations and implications, J. Phys. D: Appl. Phys. 41 (2008) 234009.

[51] M.G. McHarg, H.C. Stenbaek-Nielsen, T. Kanmae, R.K. Haaland RK, Streamer tip splitting in sprites, J. Geophys. Res. (Space Phys) 115 (2010) A00E53.

[52] S.V. Pancheshnyi, A.Y. Starikovskii, Comments on 'The role of photoionization in positive streamer dynamics', J. Phys. D: Appl. Phys. 34 (2001) 248.

[53] M. Arrayás, U. Ebert, W. Hundsdorfer, Spontaneous branching of anode-directed streamers between planar electrodes, Phys. Rev. Lett. 88 (2002) 174502.

[54] D. Bensimon, L.P. Kadanoff, S. Liang, B.I. Shraiman, C. Tang, Viscous flows in two dimensions, Rev. Mod. Phys. 58 (1986) 977.

[55] W.van Saarloos, Three basic issues concerning interface dynamics in nonequilibrium patterrn formation, Phys. Rep. 301 (1998) 9.

[56] A. Rocco, U. Ebert, and W. Hundsdorfer, Branching of negative streamers in free flight, Phys. Rev. E 66 (2002) 035102.

[57] A. A. Kulikovsky, Comment on [53], Phys. Rev. Lett. 89 (2002) 229401.

[58] U. Ebert, W. Hundsdorfer, Reply to [57], Phys. Rev. Lett. 89 (2002) 229402.

[59] C. Montijn, W. Hundsdorfer, U. Ebert, An adaptive grid refinement strategy for the simulation of negative streamers, J. Comp. Phys. 219 (2006) 801.

[60] C. Montijn, U. Ebert, W. Hundsdorfer, Numerical convergence of the branching time of negative streamers, Phys. Rev. E 73 (2006) 065401.

[61] A. Luque, U. Ebert, and W. Hundsdorfer, Interaction of streamers in air and other oxygen-nitrogen mixtures, Phys. Rev. Lett. 101 (2008) 075005.

[62] A. Luque, U. Ebert, Density models for streamer discharges: beyond cylindrical symmetry and homogeneous media, submitted to J. Comput. Phys., arXiv:1011.2871v1.

[63] U. Ebert and W. van Saarloos, Front propagation into unstable states: Universal algebraic convergence towards uniformly translating pulled fronts, Physica D 146 (2000) 1.

[64] G. Derks, U. Ebert, B. Meulenbroek, Laplacian instability of planar streamer ionization fronts an example of pulled front analysis, J. Nonlinear Sci. 18 (2008) 551-590, erratum 591-592.

[65] M. Arrayás and U. Ebert, Stability of negative ionization fronts: regularization by electric screening?, Phys. Rev. E 69 (2004) 036214.

[66] J.W. Evans, Nerve axon equations IV. The stable and unstable impulse, Indiana Univ. Math. J. 24 (1975) 1169.

[67] J. Alexander, R. Gardner, C.K.R.T. Jones, A topological invariant arising in the stability analysis of traveling waves, J. Reine Angew. Math. 410 (1990) 167.

[68] D. Terman, Stability of Planar Wave Solutions to a Combustion Model, SIAM J. Math. Anal. 21 (1990) 1139.

[69] B. Sandstede. Stability of travelling waves, in: Handbook of Dynamical Systems II (B. Fiedler, ed.). North-Holland (2002) pp. 983-1055. 
[70] T. Kapitula, B. Sandstede, Eigenvalues and resonances using the Evans function, Discrete and Continuous Dynamical Systems 10 (2004) 857.

[71] M. Arrayás, M.A. Fontelos, J.L. Trueba, Mechanism of branching in negative ionization fronts, Phys. Rev. Lett. 95 (2005) 165001.

[72] M. Arrayás, S. Betelú, M.A. Fontelos, J.L. Trueba, Fingering from ionization fronts in plasmas, SIAM J. Appl. Math. 68 (2007) 1122.

[73] U. Ebert, G. Derks, Comment on [71], Phys. Rev. Lett. 101 (2008) 139501.

[74] E.D. Lozansky, O.B. Firsov, Theory of the initial stage of streamer propagation, J. Phys. D: Appl. Phys. 6 (1973) 976.

[75] B. Meulenbroek, A. Rocco, and U. Ebert, Streamer Branching rationalized by Conformal Mapping Techniques, Phys. Rev. E 69 (2004) 067402.

[76] U. Ebert and W. van Saarloos, Breakdown of the standard Perturbation Theory and Moving Boundary Approximation for "Pulled" Fronts, Phys. Rep. 337 (2000) 139.

[77] S. Howison, Cusp development in Hele-Shaw flow with a free surface, SIAM J. Appl. Math. 46 (1986) 20.

[78] F. Brau, A. Luque, B. Meulenbroek, U. Ebert, L. Schäfer, Construction and test of a moving boundary model for negative streamer discharges, Phys. Rev. E 77 (2008) 026219.

[79] F. Brau, B. Davidovitch, U. Ebert, Moving boundary approximation for curved streamer ionization fronts: Solvability analysis, Phys. Rev. E 78 (2008) 056212.

[80] F. Brau, A. Luque, B. Davidovitch, U. Ebert, Moving boundary approximation for curved streamer ionization fronts: numerical tests, Phys. Rev. E 79 (2009) 066211.

[81] B. Meulenbroek, U. Ebert, L. Schäfer, Regularization of moving boundaries in a Laplacian field by a mixed Dirichlet-Neumann boundary condition: exact results, Phys. Rev. Lett. 95 (2005) 195004.

[82] U. Ebert, B. Meulenbroek, L. Schäfer, Convective stabilization of a Laplacian moving boundary problem with kinetic undercooling, SIAM J. Appl. Math. 68 (2007) 292.

[83] S. Tanveer, L. Schäfer, F. Brau, U. Ebert, A moving boundary problem motivated by electric breakdown: I. Spectrum of linear perturbations, Physica D 238 (2009) 888.

[84] C.-Y. Kao, F. Brau, U. Ebert, L. Schäfer, S. Tanveer, A moving boundary model motivated by electric breakdown: II. Initial value problem, Physica D 239 (2010) 1542.

[85] M. Günther, G. Prokert, On travelling-wave solutions for a moving boundary problem of Hele-Shaw type, IMA J. Appl. Math. 74 (2009) 107.

[86] P.G. Saffman and G.I. Taylor, The penetration of a fluid in a porous medium of Hele-Shaw cell containing a move viscous fluid, Proc. Roy. Soc. A 245 (1958) 312.

[87] A. Luque, F. Brau, U. Ebert, Saffman-Taylor streamers: Mutual finger interaction in spark formation, Phys. Rev. E 78 (2008) 016206.

[88] P. Pelcé, Dynamics of Curved Fronts, Academic (Boston, 1988).

[89] D. Kessler, J. Koplik, H. Levine, Pattern selection in fingered growth phenomena, Adv. Phys. 37 (1988) 255.

[90] S. Tanveer, Surprises in viscous fingering, J. Fluid Mech. 409 (2000) 273.

[91] S.J. Chapman, J.R. King, The selection of Saffman-Taylor fingers by kinetic undercooling, J. of Eng. Math. 46 (2003) 1.

[92] S. Tanveer, New solutions for steady bubbles in a Hele-Shaw cell, Phys. Fluids 30 (1987) 651.

[93] R. Combescot, T. Dombre, Selection in the Saffman-Taylor bubble and asymmetrical finger problem, Phys. Rev. A 38 (1988) 2573. 


\section{Figure caption for the title page of Nonlinearity 2011:}

Feather-like structures in a positive streamer discharge in pure argon at room temperature and atmospheric pressure. The discharge is generated by applying a 22 $\mathrm{kV}$ voltage pulse of microsecond duration on a sharp tungsten tip located $160 \mathrm{~mm}$ above a grounded plate. The image is recorded with a fast ICCD camera and represents about $40 \mathrm{~mm}$ of the discharge gap with the electrode tip in the top center. It was published originally in Fig. 7 of [1] and is also shown with more density levels in the article accompanying the cover page. 


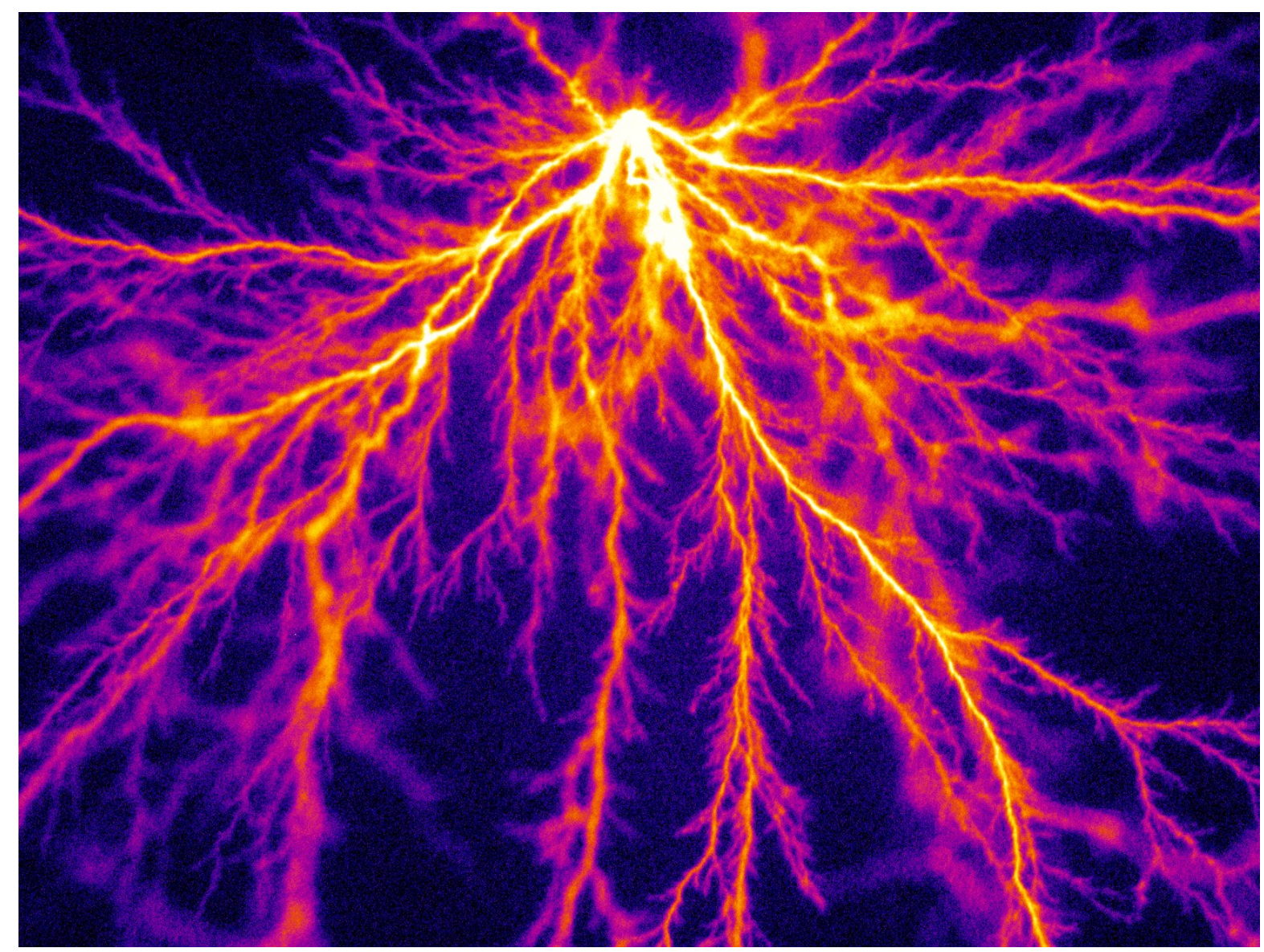

Figure 1. Feather-like structures in a positive streamer discharge in pure argon at room temperature and atmospheric pressure (in false colours). The discharge is generated by applying a $22 \mathrm{kV}$ voltage pulse of microsecond duration on a sharp tungsten tip located $160 \mathrm{~mm}$ above a grounded plate. The image is recorded with a fast ICCD camera and represents about $40 \mathrm{~mm}$ of the discharge gap with the electrode tip in the top center. The blurred structures are out of focus. The figure was published originally in Fig. 7 of [1]; it appears also on the cover of Nonlinearity in 2011. 

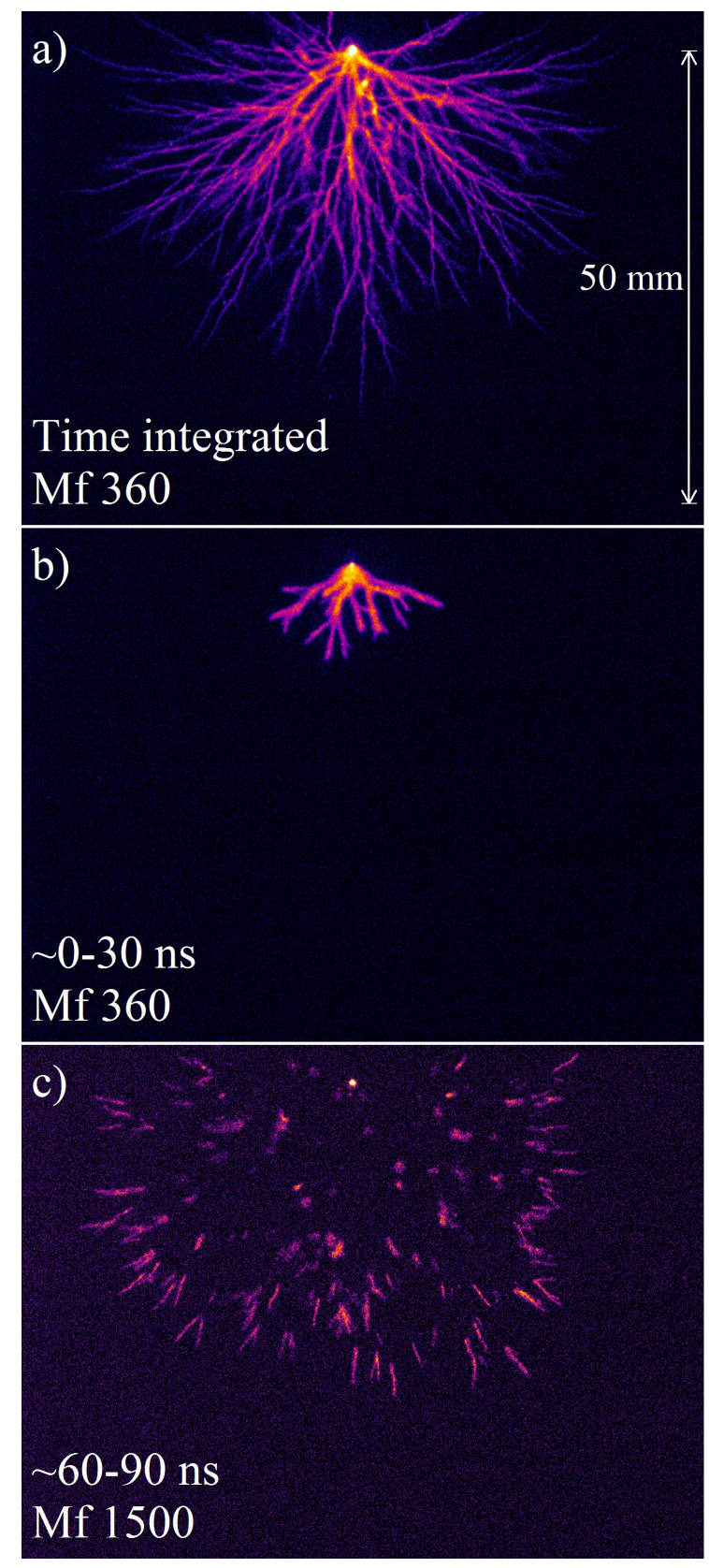

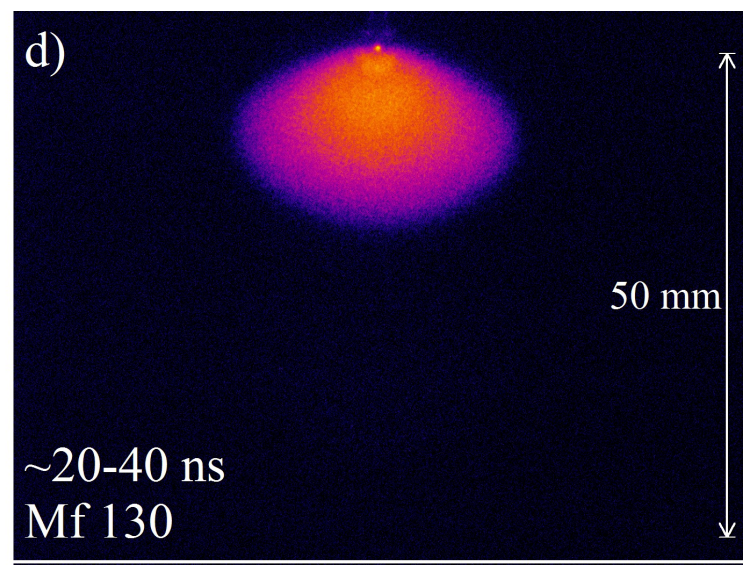

e)
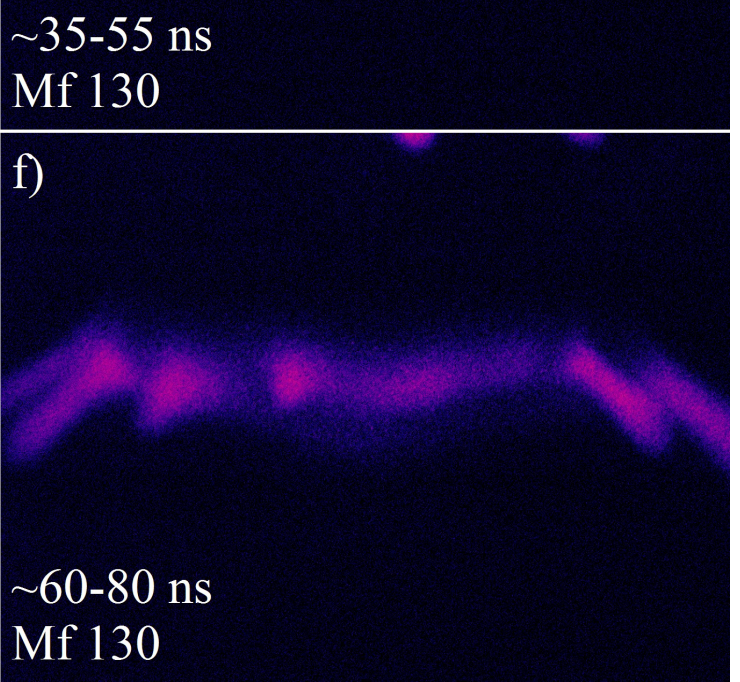

Figure 2. Positive discharges in artificial air $\left(\mathrm{N}_{2}: \mathrm{O}_{2}=80: 20\right)$ at room temperature. A voltage pulse of $35 \mathrm{kV}$ and of $130 \mathrm{~ns}$ duration is applied to the upper needle electrode (with the Blumlein pulser described in [1]), the distance to the plate electrode below is $16 \mathrm{~cm}$. Only the upper $5 \mathrm{~cm}$ of the gap are shown. Left column: A positive streamer discharge tree at atmospheric pressure. (a): Time integrated picture; (b): light emission during the early time interval [0 ns, $30 \mathrm{~ns}]$; (c): light emission during the late time interval [60 ns, $90 \mathrm{~ns}$ ]. Right column: A zoom into the formation of positive streamers. As the pressure in (d-f) is 200 mbar, i.e., 1/5 of the pressure in (a-c), all length and time scales are 5 times larger according to the Townsend scaling explained in sections 1.2 and 2.1. (d): glowing ball next to the electrode at time interval [20 ns, $40 \mathrm{~ns}$ ]; (e): destabilizing shell or front at time interval [35 ns, $55 \mathrm{~ns}$; (f): destabilization of the front into streamers at time interval [60 ns, $80 \mathrm{~ns}$ ]. Panel (b) can be seen as the next stage of evolution after (f). The Mf-value measures the gain of the camera system and the false color scale as defined in [1]; a higher value indicates more gain and thus a dimmer discharge. Note that only one picture per discharge can be taken; therefore different pictures are from similar, but not identical events. The indicated time intervals are relative to the start of the voltage pulse, although there can be an error of order 10-20 ns in the exact positioning of the 0 ns moment. 


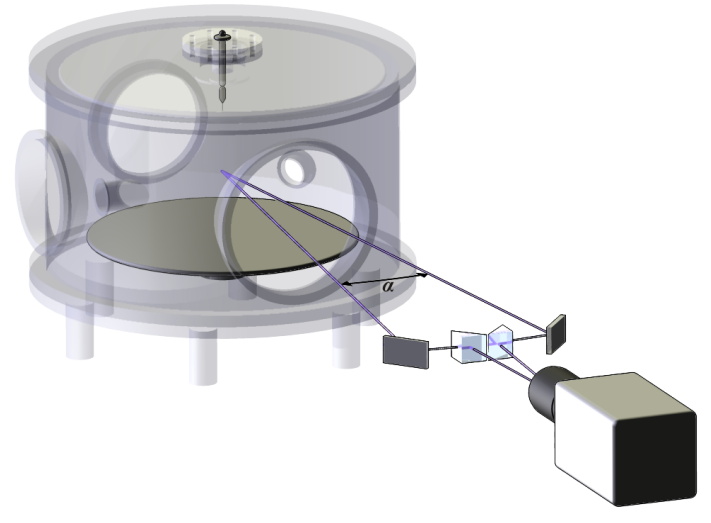

(a) Set-up for stereographic imaging.

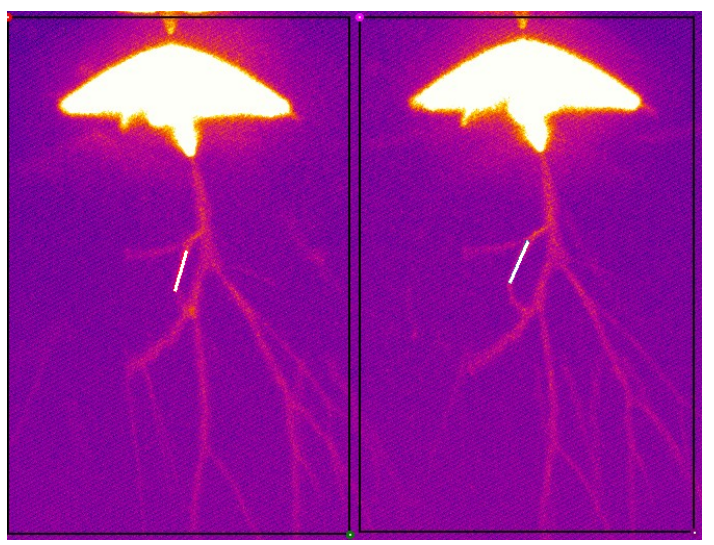

(b) The two resulting images, taken at a relative angle of $14^{\circ}$.

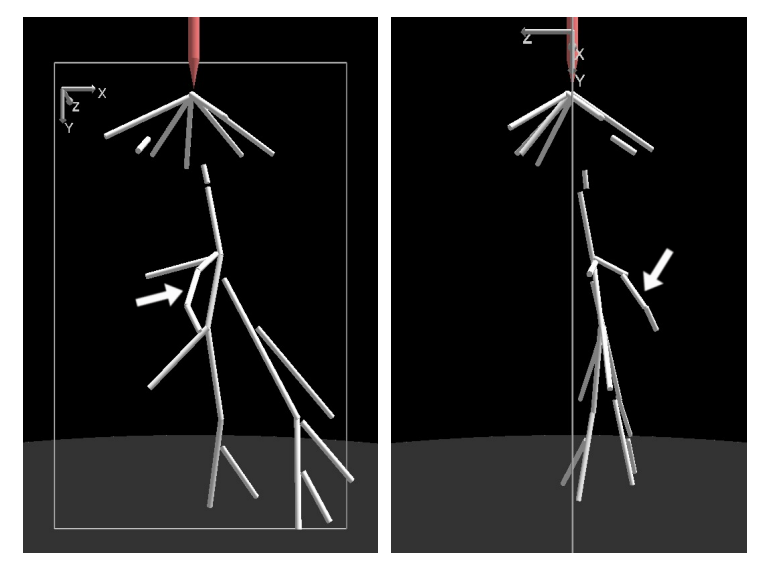

(c) Orthogonal views of the $3 \mathrm{D}$ reconstruction. The section originally marked with the white line is now marked with an arrow in both views.

Figure 3. 3D reconstruction of branching positive streamers in ambient air at room temperature and a pressure of 200 mbar, reproduced from [18]. The pulsed voltage is $U=47 \mathrm{kV}$. The distance from needle electrode above to plate electrode below is $14 \mathrm{~cm}$. 


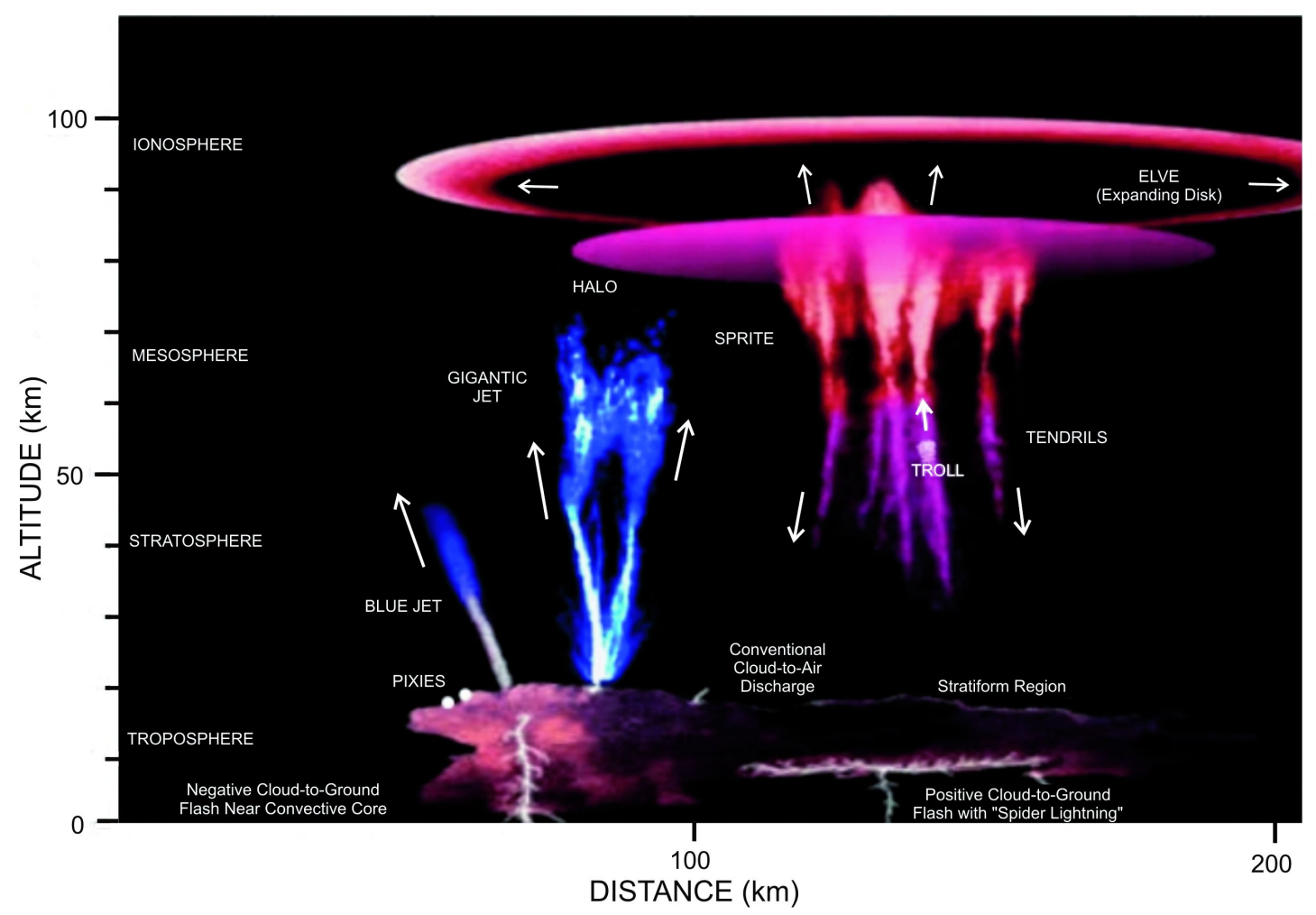

Figure 4. The zoo of transient luminous events generated by thunderstorms above the clouds. Most names are taken from Shakespeare's Midsummer Night's Dream. Elves and sprites are the most frequent. Ground and ionosphere can be considered as equipotential; the thundercloud is the voltage supply separating electrical charges. The break-down electric field is proportional to air density; therefore it decreases strongly with altitude. [Fig. by D.D. Sentman, Univ. Alaska in Fairbanks.] 

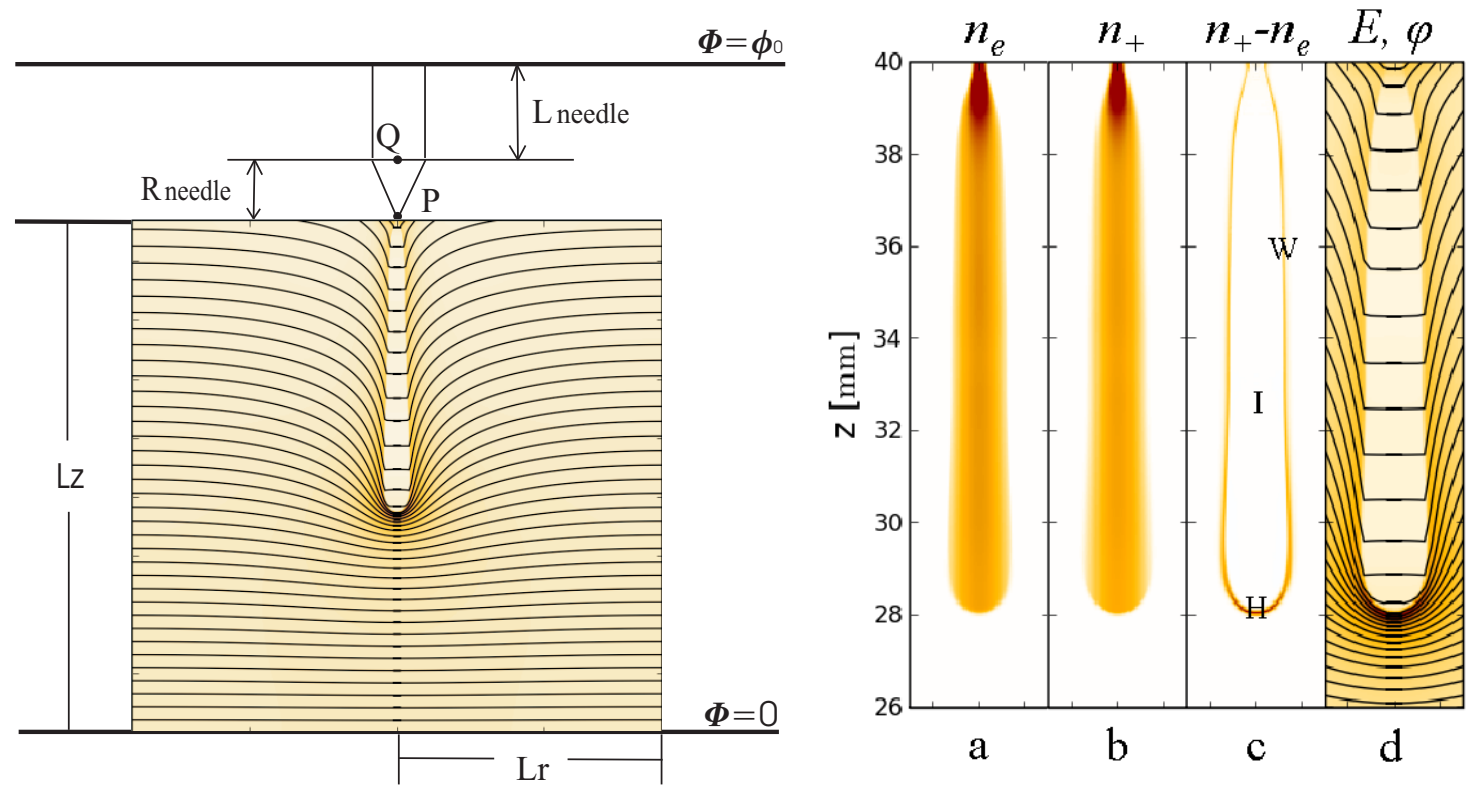

Figure 5. Simulation of a positive streamer in air (i.e., with nonlocal photo-ionization) that propagates from a needle electrode downwards. The applied voltage is $55 \mathrm{kV}$, the distance from needle electrode above to plate electrode below is $4 \mathrm{~cm}$, i.e., the average field is $14 \mathrm{kV} / \mathrm{cm}$ or 0.07 in our dimensionless units. Left: Electrode configuration with electric field strength (colour coded) and equipotential lines. The electric field is calculated in the whole region between the planar electrodes, and the particle densities in the shaded region below the needle. Right: Streamer structure shown by zooming into the relevant region. The panels show: a) electron density $n_{e}$, b) ion density $n_{+}$, c) space charge density $q=e\left(n_{+}-n_{e}\right)$, d) electric field strength $E$ (color coded) and equipotential lines $\varphi$. The letters in panel c indicate the streamer regions: $\mathrm{H}$ - streamer head, I - interior and $\mathrm{W}$ - wall of the streamer channel. The figures are taken from [26] where details can be found. 

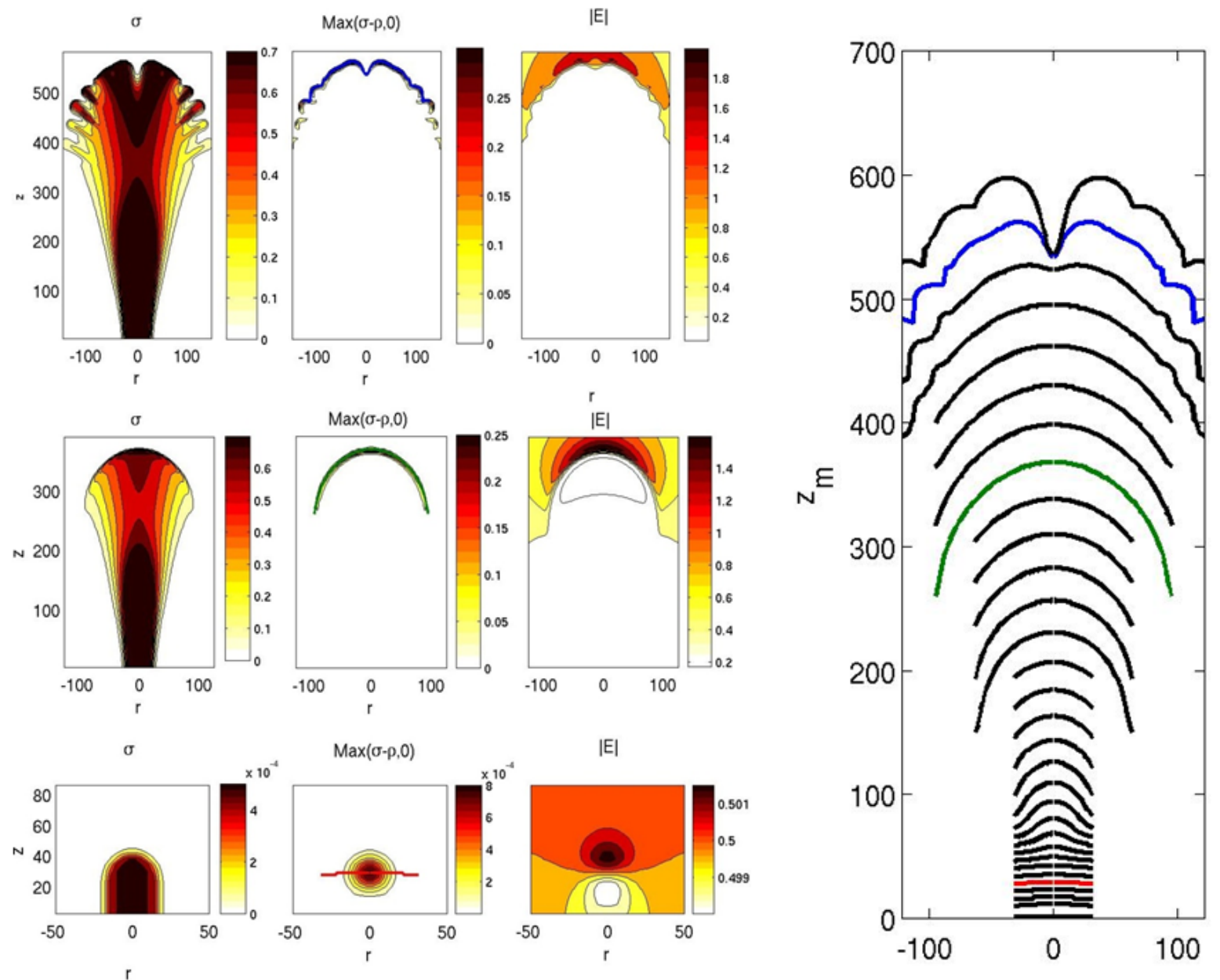

Figure 6. The $3 \times 3$ panels on the left: From bottom to top: Evolution of a negative discharge in nitrogen (hence without nonlocal photo-ionization). The background field is 0.5 in dimensionless units. The earliest stage is the initial avalanche (lower row), the next the propagating streamer (middle row), the latest the branching streamer (with a constraint of cylindrical symmetry, see text). From left to right: dimensionless electron density $\sigma$, dimensionless negative space charge density $\sigma-\rho$ with $\max _{r}(\sigma-\rho)(r, z)$ indicated by a colored line, and the absolute value of the dimensionless electric field. Right: The contours of $\max _{r}(\sigma-\rho)(r, z)$ in regular time steps; the colored profiles from the middle column of the left figure block are indicated here in the same colors. As in Fig. 5 (right), the figures zoom into the streamer structure while the total simulation volume is much larger. The calculations are performed on adaptively refined numerical grids and are described in detail in [59]. 


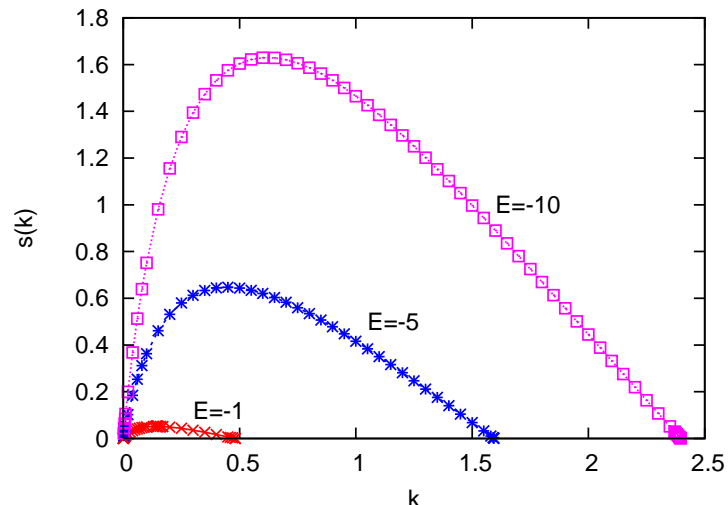

(a) $E_{\infty}=-1,-5$ and -10 and fixed $D=0.1$.

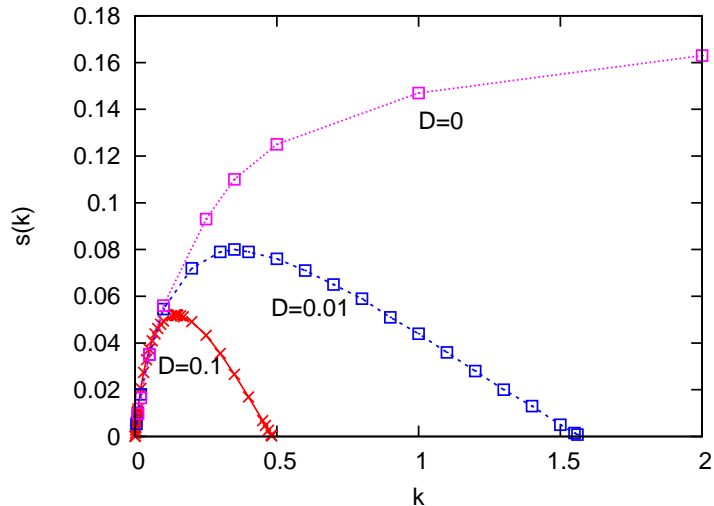

(b) Fixed $E_{\infty}=-1$ and $D=0.1,0.01$ and 0 .

Figure 7. Dispersion curves $s(k)$ : (a) for varying $E_{\infty}$ and fixed $D=0.1$, and (b) for fixed $E_{\infty}=-1$ and varying $D$. The data for the singular limit $D=0$ are taken from [65]. The figures are reproduced from [64].

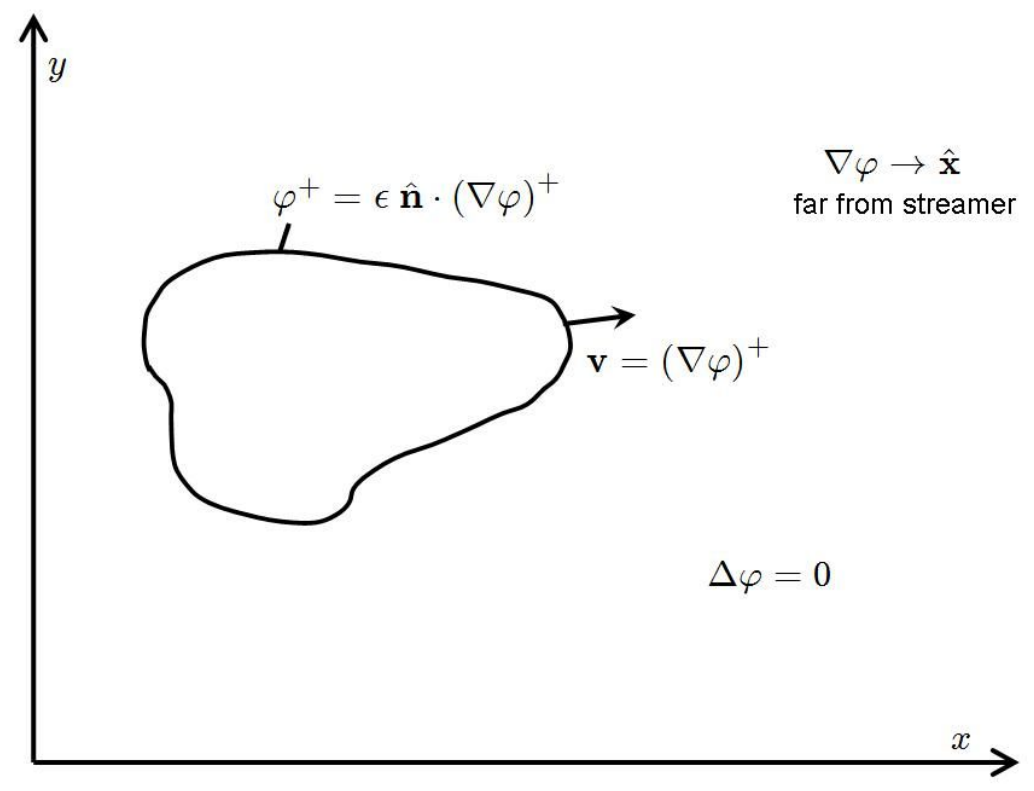

Figure 8. Illustration of the model problem in terms of the Laplace field $\varphi$. 


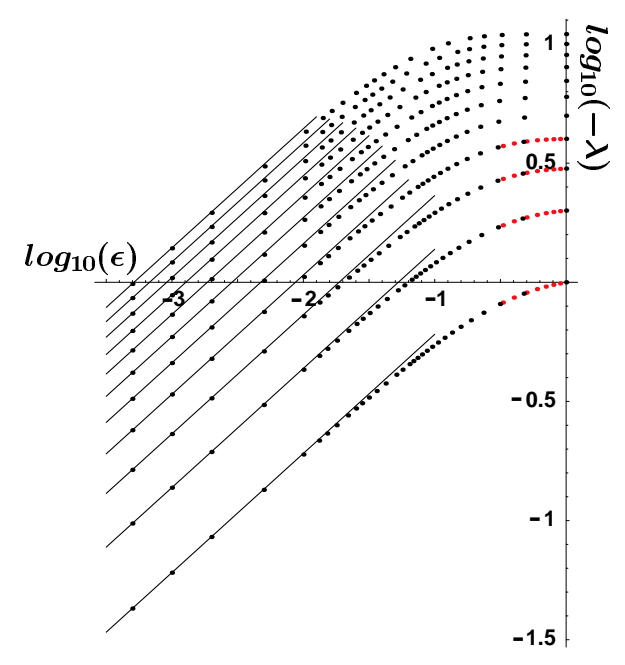

Figure 9. Double logarithmic plot of the eigenvalues $\lambda_{n}(\epsilon), n=1, \ldots, 11$ (from bottom to top) as a function of $\epsilon$ in the range $5 \cdot 10^{-4} \leq \epsilon \leq 1$. Black or red dots indicate eigenvalues calculated with different methods. The lines give the asymptotic behaviour for $\epsilon \rightarrow 0$. Details can be found in [83] where the present figure appears as Fig. 1.

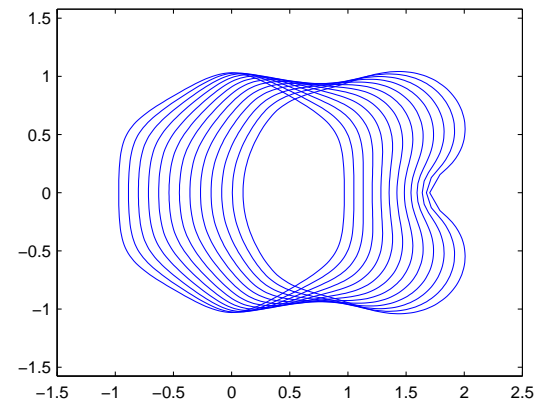

(a) $\epsilon=1 / 10$, nonlinear

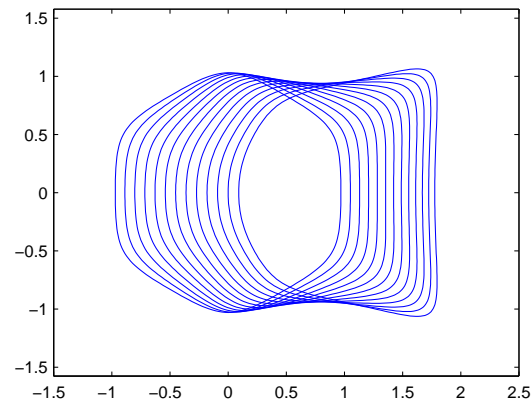

(b) $\epsilon=1 / 10$, linear

Figure 10. The evolution of a convecting "streamer" circle in two dimensions with initial perturbation $\beta(\omega, 0)=-0.03 \omega^{5}$. (a) and (b) show the nonlinear and the linear evolution respectively for regularization parameter $\epsilon=1 / 10$. Details of the solution strategy can be found in [84] where the present figure appears as Fig. 12. 


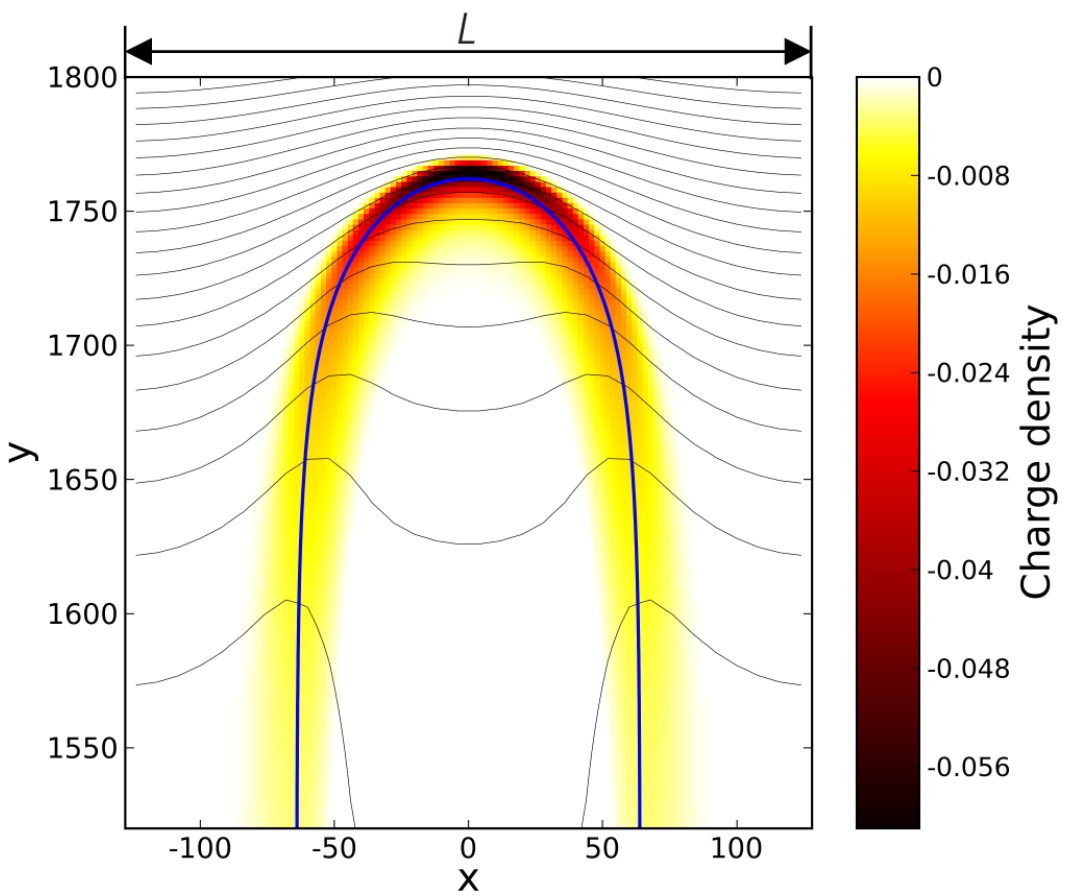

Figure 11. Color coded: negative charge density $\sigma-\rho$ of the minimal streamer model in two dimensions with a Neumann boundary condition for the electric potential on the lateral boundaries. This lateral boundary is equivalent to a symmetry plane between two streamers, hence the distance from one streamer center to the next is $L$. Thin black lines: lines of equal electric potential $\varphi$. Thick blue line: The selected solution (37) of the Saffman-Taylor-problem of two-fluid flow. The figure is reproduced from Fig. 3 in [87] where details can be found. 